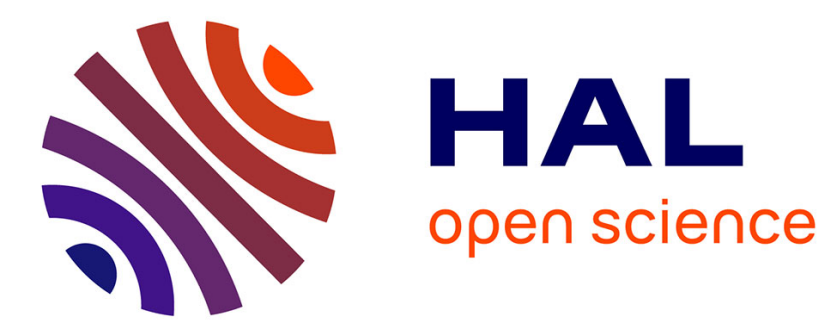

\title{
On the conicity of eigenvalues intersections for parameter-dependent self-adjoint operators
}

Francesca Chittaro, Paolo Mason

\section{To cite this version:}

Francesca Chittaro, Paolo Mason. On the conicity of eigenvalues intersections for parameter-dependent self-adjoint operators. Journal of Mathematical Physics, 2020, 61 (5), 10.1063/1.5115576 . hal02160192v2

\section{HAL Id: hal-02160192 \\ https://hal.science/hal-02160192v2}

Submitted on 14 May 2020

HAL is a multi-disciplinary open access archive for the deposit and dissemination of scientific research documents, whether they are published or not. The documents may come from teaching and research institutions in France or abroad, or from public or private research centers.
L'archive ouverte pluridisciplinaire HAL, est destinée au dépôt et à la diffusion de documents scientifiques de niveau recherche, publiés ou non, émanant des établissements d'enseignement et de recherche français ou étrangers, des laboratoires publics ou privés. 
Conicity of eigenvalues intersections

On the conicity of eigenvalues intersections for parameter-dependent self-adjoint operators

Francesca Carlotta Chittaro ${ }^{1, \text { a) }}$ and Paolo Mason ${ }^{2, b)}$

1) Université de Toulon, Aix Marseille Univ, CNRS, LIS, Marseille, France.

${ }^{2)}$ Laboratoire des Signaux et Systèmes (L2S, UMR 8506), CNRS - CentraleSupelec - Université Paris-Sud, 3, rue Joliot Curie, 91192, Gif-sur-Yvette, France

(Dated: 20 February 2020)

Motivated by recent controllability results for the bilinear Schrödinger equation based on the existence of conical intersections, in this paper we identify two physically interesting families of parameter-dependent Hamiltonians that admit residual and prevalent subfamilies for which all double eigenvalues are conical. In order to obtain such a result we exploit a characterization of conical intersections in terms of a transversality condition which allows to apply a suitable transversality theorem.

\footnotetext{
a) Electronic mail: francesca.chittaro@univ-tln.fr

b)Electronic mail: paolo.mason@12s.centralesupelec.fr
} 


\section{INTRODUCTION}

Consider a family of Hermitian matrices, smoothly depending on a finite number of parameters; it is well known that the corresponding eigenvalues are smooth real functions of these parameters. It may happen that the graphs of some of these functions intersect; since intersection points carry important topological information, and are often associated with many physically interesting phenomena, they have attracted great interest in both the mathematics and the physics community, in particular for what concerns their classification and the problem of their occurrence and detection ${ }^{3,11-13,16,31,33}$. This kind of problems have also been addressed in the general case of self-adjoint operators acting on separable Hilbert spaces $^{5,22,32}$.

In mathematical physics, a special role is played by conical intersections (also known as generic coalescing points, diabolic points), which may be encountered in several applications in molecular dynamics, physical chemistry, solid-state physics (e.g. the well-known Diracpoints in graphene), optics (e.g. systems of ultracold Fermi atoms in optical lattices). For an account of several applications related to conical intersections, see e.g. Ref. 14 and references therein.

Recently, conical intersections have been proved to be useful in control theory, namely for the population transfer problem and controllability issues concerning bilinear Schrödinger equations $^{2,4,6,7,9}$, that is, equations of the form

$$
i \frac{\partial \psi}{\partial t}=\left(H_{0}+\sum_{k=1}^{m} u_{k} H_{k}\right) \psi,
$$

where $\psi$ is a unit vector in some separable complex Hilbert space $\mathcal{H}, H_{i}$ are self-adjoint operators on $\mathcal{H}$ for $i=0, \ldots, m$, and $H\left(u_{1}, \ldots, u_{m}\right)=H_{0}+\sum_{k=1}^{m} u_{k} H_{k}$ is the control-dependent (or parameter-dependent) Hamiltonian. By following the same approach, controllability results for nonlinear control-dependent Hamiltonians have been obtained in Ref. 8.

It is worth asking if eigenvalues intersections are "typically" conical, or if this feature is "pathological". In other words, given a family of parameter-dependent self-adjoint operators, we wish to investigate if "almost every" element in this family has only conical eigenvalues intersections. This problem is well defined in a finite dimensional setting, as it is possible to endow the family with a natural measure; in this case, one has to check whether the set of parameter-dependent self-adjoint operators admitting only conical intersections has full 
measure. In an infinite dimensional setting, in absence of an appropriate choice of measure, a natural generalization of the concept of full measure set still appears to be possible: it is the notion of prevalent set ${ }^{17}$, roughly speaking a set such that each of its translations has full measure, for some compactly supported measure. Alternatively, one may consider some topological notion replacing the property of being full-measure: a subset of a Baire space $X$ is said to be residual if it contains a countable intersection of open and dense subsets of $X$, while a generic property is a property that holds on a residual subset of $X$. It is worth noting that, although the latter notion is much more used in the literature to describe properties that hold "almost everywhere" on functional spaces, in a finite dimensional space residual sets are not necessarily of full measure and, vice versa, it is possible to exhibit full measure sets which are not residual.

In order to determine if eigenvalues intersections are conical for "almost every" parameterdependent self-adjoint operators (in the previous senses), we first establish some criteria for a double eigenvalue to be conical. For a self-adjoint operator $H(\cdot)$ smoothly depending on three real parameters, the conicity of an eigenvalues intersection is equivalent to the nondegeneracy of a three-dimensional matrix, which depends on the eigenstates corresponding to the degenerate eigenvalue and on the linearization of the operator with respect to the parameters; this result is proved in Proposition IV.3. Furthermore, whenever the parameterdependent self-adjoint operator takes values in a Banach manifold, the conicity of an eigenvalues intersection can be characterized (roughly speaking) in terms of the transversality of $H(\cdot)$ to the submanifold of self-adjoint operators with multiple eigenvalues (Proposition V.1). The issue of "measuring" the set of parameter-dependent Hamiltonians having only conical intersections is thus translated in the problem of measuring the set where the map $H(\cdot)$ is transversal to that manifold. Powerful tools to obtain this kind of results are the Transversal Density Theorem ${ }^{1}$ and the Parametric Transversality Theorem ${ }^{21}$ : they provide sufficient conditions that guarantee that the set of points where a map is transversal is, respectively, residual (on a Banach manifold) or of full-measure (on a finite dimensional manifold). An analogous result ${ }^{18}$ can be used to establish properties that hold true on prevalent subsets of (possibly) infinite dimensional Banach manifolds.

We focus on two physically interesting cases. In Section VA, we consider control-affine Hermitian matrices; we prove that, for every fixed $n$-dimensional Hermitian matrix $H_{0}$, the set of triples $\left(H_{1}, H_{2}, H_{3}\right) \in(i \mathfrak{u}(n))^{3}$ such that all double eigenvalues of the parameter- 
dependent operator $H_{0}+\sum_{j=1}^{3} u_{j} H_{j}$ correspond to conical intersections is residual and of full Lebesgue measure. In Section VB we are concerned with operators of the form $\left(-i \nabla+u_{3} \mathbf{A}\right)^{2}+u_{1} V_{1}+u_{2} V_{2}$, that represent the Hamiltonians of particles in a controlled electromagnetic field. We prove that, generically with respect to the control operators A, $V_{1}, V_{2}$, all double eigenvalues are conical, and the corresponding set of control operators is prevalent.

Note that classical transversality results require the space where such operators live to be second countable. This property is usually not satisfied for spaces of linear operators acting on infinite dimensional spaces (even for the space of bounded operators on an Hilbert space). Thus, checking the genericity or the prevalence on larger operator spaces, compared to the physically interesting ones, is not only pointless (for a subset of a Banach manifold, the property of being residual or prevalent is not preserved if one restricts to a submanifold) but also technically more involved.

We finally remark that in this paper we only consider the three-input case. For the twoinput case, if all the operators in the family have real-valued components with respect to a fixed basis, the genericity of conical intersections is investigated in Ref. 6. Otherwise, if the operator does not depend on more than two control inputs, eigenvalues intersections are usually absent as a consequence of the fact that the set of operators admitting (double) eigenvalues intersections is a manifold of codimension three in the space of self-adjoint operators (see e.g. Refs. 3 and 33). In addition, it is easy to see with the methods developed in this paper that, when more than three controls are available, eigenvalues intersections are generically not isolated (hence not conical).

The structure of the paper is the following: in Section II, we state the notations we are using throughout the paper; in Section III, we introduce the notions of genericity and prevalence and state the problem we are concerned with; Section IV is devoted to the definition and the main properties of conical intersections; finally, the main results and their proofs are provided in Section V. Some technical results on the regularity properties of perturbed self-adjoint operators are illustrated in Appendix A. 


\section{NOTATIONS AND GENERAL SETTING}

We use bold letters (e.g., u) to denote elements of $\mathbb{R}^{3}$, and regular fonts for the corresponding components (e.g., $u_{i}$ for the $i$-th component of $\mathbf{u}$ ). Throughout this paper, we consider operators acting on a separable complex Hilbert space $\mathcal{H}$, with scalar product $\langle\cdot, \cdot\rangle$ and norm $\|\cdot\|$. To avoid possible ambiguities, we denote the Euclidean norm on finite dimensional spaces other than $\mathcal{H}$ by $|\cdot|$. The set of the linear operators on $\mathcal{H}$ is denoted by $\mathcal{L}(\mathcal{H})$; the subspace $\mathcal{B}(\mathcal{H})$ of bounded operators in $\mathcal{L}(\mathcal{H})$ is endowed with the operator norm induced from the one of $\mathcal{H}$, still denoted as $\|\cdot\|$. Given a linear operator $A \in \mathcal{L}(\mathcal{H}), \mathcal{D}(A)$ denotes its domain; $\mathcal{D}(A)$ is naturally endowed with the norm of the graph of $A$, defined as

$$
\|\psi\|_{\mathcal{D}(A)}=\|A \psi\|+\|\psi\|, \quad \psi \in \mathcal{D}(A) .
$$

We introduce the following notions of "relative boundedness" between linear operators in $\mathcal{H}$.

Definition II.1 ( $A$-smallness and $A$-boundedness) Let $A$ and $B$ be two densely defined operators with domains $\mathcal{D}(A) \subset \mathcal{D}(B)$. We say that $B$ is $A$-bounded if there exist $a, b>0$ such that $\|B \psi\| \leq a\|A \psi\|+b\|\psi\|$ for every $\psi \in \mathcal{D}(A)$. B is said to be $A$-small if for every $\alpha>0$ there exists $\beta>0$ such that $\|B \psi\| \leq \alpha\|A \psi\|+\beta\|\psi\|$ for every $\psi \in \mathcal{D}(A)$.

These notions are also referred to as Kato boundedness and Kato smallness or infinitesimal smallness with respect to $A$, see e.g. Ref. 27.

Given a self-adjoint operator $A$ on $\mathcal{H}$, we denote the space of $A$-bounded operators, which coincide with the continuous linear operators from $\mathcal{D}(A)$ to $\mathcal{H}$, by $\mathcal{B}(\mathcal{D}(A), \mathcal{H})$. We endow the space of $A$-bounded operators with the operator norm from $\mathcal{D}(A)$ to $\mathcal{H}$, denoted as

$$
\|B\|_{A}=\sup _{\psi \in \mathcal{D}(A)} \frac{\|B \psi\|}{\|\psi\|_{\mathcal{D}(A)}}
$$

for every $A$-bounded operator $B$. The subspace of self-adjoint operators in $\mathcal{B}(\mathcal{D}(A), \mathcal{H})$ is denoted by $\mathcal{B}_{s a}(\mathcal{D}(A), \mathcal{H})$. Similarly, $\mathcal{B}(\mathcal{H}, \mathcal{D}(A))$ denotes the space of continuous linear operators from $\mathcal{H}$ to $\mathcal{D}(A)$. The natural norm on this space $\|\cdot\|_{\mathcal{B}(\mathcal{H}, \mathcal{D}(A))}$ is equivalent to the norm $\|A \cdot\|+\|\cdot\|$, therefore, with a little abuse of notation, in the following we set

$$
\|B\|_{\mathcal{B}(\mathcal{H}, \mathcal{D}(A))}=\|A B\|+\|B\| .
$$


$\mathcal{B}_{\text {sa }}(\mathcal{H}, \mathcal{D}(A))$ is the subspace of self-adjoint operators contained in $\mathcal{B}(\mathcal{H}, \mathcal{D}(A))$.

In this paper, we are concerned with families of $\mathcal{C}^{1}$ operator-valued functions ${ }^{23}$ (parameterdependent Hamiltonians) $H: \mathbb{R}^{3} \rightarrow \mathcal{B}_{s a}(\mathcal{D}(H(0)), \mathcal{H})$ such that $H(\mathbf{u})-H(0)$ is $H(0)$-small. In particular, for every $\overline{\mathbf{u}} \in \mathbb{R}^{3}$,

$$
\begin{aligned}
\lim _{\mathbf{u} \rightarrow \overline{\mathbf{u}}}\|H(\mathbf{u})-H(\overline{\mathbf{u}})\|_{H(0)} & =0, \\
\lim _{\mathbf{u} \rightarrow \overline{\mathbf{u}}}\left\|\partial_{i} H(\mathbf{u})-\partial_{i} H(\overline{\mathbf{u}})\right\|_{H(0)} & =0, \quad i=1,2,3,
\end{aligned}
$$

where here and in the following $\partial_{i}$ denotes the derivative with respect to $u_{i}, i=1,2,3$. It is easy to see that, under this assumption, the norms $\|\cdot\|_{H(\mathbf{u})}$ are equivalent for every $\mathbf{u} \in \mathbb{R}^{3}$, namely there exist two continuous positive functions $C_{1}, C_{2}: \mathbb{R}^{3} \rightarrow \mathbb{R}$ such that

$$
C_{1}(\mathbf{u})\|\cdot\|_{H(\mathbf{u})} \leq\|\cdot\|_{H(0)} \leq C_{2}(\mathbf{u})\|\cdot\|_{H(\mathbf{u})} .
$$

Remark II.2 Any operator-valued function $H(\cdot)$ polynomial with respect to its argument satisfies the assumption here above, provided that all its coefficients are self-adjoint and H(0)-small operators. This is a simple consequence of Ref. 27, Theorem X.12.

Under these assumptions, the spectrum and the spectral projections of $H(\mathbf{u})$ possess some regularity properties with respect to $\mathbf{u}$, which will be discussed in Section A.

\section{GENERICITY AND PREVALENCE FOR CONICAL INTERSECTIONS: STATEMENT OF THE PROBLEM}

Here below, $\sigma(H(\mathbf{u}))$ denotes the spectrum of the operator $H(\mathbf{u})$. We say that two (or more) eigenvalues intersect at $\overline{\mathbf{u}} \in \mathbb{R}^{3}$, and that $\overline{\mathbf{u}}$ is an eigenvalues intersection, if their values coincide at $\overline{\mathbf{u}}$. In particular, we are interested in conical intersections, which are defined as follows (see also Refs. 6 and 9$)^{24}$.

Definition III.1 Consider a $\mathcal{C}^{1}$ operator-valued function $H: \mathbb{R}^{3} \rightarrow \mathcal{B}_{\text {sa }}(\mathcal{D}(H(0)), \mathcal{H})$ such that $H(\mathbf{u})-H(0)$ is $H(0)$-small. We say that $\overline{\mathbf{u}} \in \mathbb{R}^{3}$ is a conical intersection between two eigenvalues if there exist an open neighborhood $U \subset \mathbb{R}^{3}$ of $\overline{\mathbf{u}}$, an interval $I \subset \mathbb{R}$ and two continuous eigenvalues $\lambda_{ \pm}: U \rightarrow \mathbb{R}$ such that $\lambda_{-}(\overline{\mathbf{u}})=\lambda_{+}(\overline{\mathbf{u}}), \sigma(H(\mathbf{u})) \cap I=\left\{\lambda_{-}(\mathbf{u}), \lambda_{+}(\mathbf{u})\right\}$, and there exists a constant $c>0$ such that, for any unit vector $\mathbf{v} \in \mathbb{R}^{3}$ and $t>0$ small 
Conicity of eigenvalues intersections

enough, we have that

$$
\lambda_{+}(\overline{\mathbf{u}}+t \mathbf{v})-\lambda_{-}(\overline{\mathbf{u}}+t \mathbf{v})>c t
$$

To measure the possibility that double eigenvalues intersections are conical we will make use of the notions of prevalent and residual set given below ${ }^{1,17}$.

Definition III.2 Let $\mathcal{A}$ be a Banach space and $S \subset \mathcal{A}$.

- We say that $S$ is prevalent in $\mathcal{A}$ if there exists a nontrivial measure defined on the Borel sets of $\mathcal{A}$ and supported on a compact subset of $\mathcal{A}$ such that all translations $a+S$, for $a \in \mathcal{A}$, are of full measure.

- We say that $S$ is residual in $\mathcal{A}$ if it contains the union of a countable family of open and dense subsets of $\mathcal{A} .^{25} A$ property which is satisfied on a residual subset of $\mathcal{A}$ is said to be generic in $\mathcal{A}$.

It has been shown in Ref. 17, Fact 6 that a subset of a finite-dimensional space is prevalent if and only if it is of full Lebesgue measure. Let us also remark that prevalence and genericity are independent notions; for instance, given a sequence of open and dense subsets of $\mathbb{R}$ whose Lebesgue measures tend to zero, their intersection is residual but not prevalent (having zero Lebesgue measure), while its complement is prevalent but not residual. Finally, we recall that countable intersections of prevalent (resp. residual) subsets of $\mathcal{A}$ are prevalent (resp. residual).

Typical problems we are concerned with in this paper are of the following type.

Problem. Let $\mathcal{A}$ be a Banach space and consider a family

$$
\left\{H_{a}: \mathbb{R}^{3} \rightarrow \mathcal{B}_{s a}\left(\mathcal{D}\left(H_{0}(0)\right), \mathcal{H}\right): a \in \mathcal{A}\right\}
$$

of parameter-dependent self-adjoint operators such that $H_{a}(\cdot)$ is $\mathcal{C}^{1}$ for every a $\in \mathcal{A}$ and $H_{a}(\mathbf{u})-H_{0}(0)$ is $H_{0}(0)$-small for every pair $(a, \mathbf{u}) \in \mathcal{A} \times \mathbb{R}^{3}$. Is it true that there exists a residual and/or prevalent subset $S$ of $\mathcal{A}$ such that, for a belonging to $S$, all double eigenvalues intersections of the operator $H_{a}(\cdot)$ are conical?

To study this problem we will take advantage of transversal density results. 
Definition III.3 Let $X, Y$ be $\mathcal{C}^{1}$ Banach manifolds (that is, manifolds modeled on Banach spaces) and $W$ a submanifold of $Y$. We say that a $\mathcal{C}^{1}$ function $f: X \rightarrow Y$ is transversal to $W$ at $x \in X$ if either $f(x) \notin W$ or $f(x) \in W$ and

(i) the inverse image $\left(f_{x}^{\prime}\right)^{-1}\left(T_{f(x)} W\right)$ splits in $T_{x} X$, that is, it is closed and it admits a closed complement in $T_{x} X$,

(ii) the image $f_{x}^{\prime}\left(T_{x} X\right)$ contains a closed complement to $T_{f(x)} W$ in $T_{f(x)} Y$.

If $f$ is transversal to $W$ at any $x \in X$, then we simply say that $f$ is transversal to $W$.

In Ref. 18, the author has shown that the assumptions of the classical Transversal Density Theorem (Ref. 1, Theorem 19.1), guaranteeing that a given (transversality) property is generic, also ensure that such a property is satisfied on a prevalent set. In a finite dimensional setting this fact actually corresponds to the well-known Parametric Transversality Theorem (Ref. 21, Theorem 6.35). These results are resumed below in a setting suitable for our purposes.

Theorem III.4 $\left(^{1,18}\right)$ Let $\mathcal{A}, Y$ be $\mathcal{C}^{1}$ Banach manifolds, $U \subset \mathbb{R}^{3}$ open and ev $: \mathcal{A} \times U \rightarrow Y$ be a $\mathcal{C}^{1}$ map. Let $W$ be a $\mathcal{C}^{1}$ submanifold of $Y$. Assume that:

- W has codimension three in $Y$;

- $\mathcal{A}$ is second countable;

- the map ev is transversal to $W$.

Then the set $\mathcal{A}_{W}$ of elements $a \in \mathcal{A}$ such that $\operatorname{ev}(a, \cdot)$ is transversal to $W$ is residual and prevalent in $\mathcal{A}$.

In Section V we will show that, under suitable assumptions, the conicity of an eigenvalues intersection is equivalent to the transversality of the map $H_{a}(\cdot)$ to a certain manifold $W$ of codimension three. In view of Theorem III.4, and provided that $\mathcal{A}$ is second countable, a positive answer to the problem stated above may thus be obtained by checking the transversality of $(a, \mathbf{u}) \mapsto H_{a}(\mathbf{u})$ to $W$. See Sections VA and VB for the application of this scheme to physically interesting examples. 


\section{PROPERTIES OF CONICAL INTERSECTIONS}

Conical intersections have a characterization in terms of the nondegeneracy of a particular matrix, that we call the conicity matrix, whose definition is given here below. A similar characterization has been obtained in Ref. 13, Theorem 4.5.

Definition IV.1 Let $H: \mathbb{R}^{3} \rightarrow \mathcal{B}_{\text {sa }}(\mathcal{D}(H(0)), \mathcal{H})$ be a $\mathcal{C}^{1}$ map such that $H(\mathbf{u})-H(0)$ is $H(0)$-small for every $\mathbf{u}$. Given two orthonormal elements $\psi_{1}, \psi_{2} \in \mathcal{D}(H(0))$, the conicity matrix of $H(\cdot)$ at $\mathbf{u} \in \mathbb{R}^{3}$ associated with $\psi_{1}, \psi_{2}$ is defined as

$$
\mathcal{M}_{\mathbf{u}}\left(\psi_{1}, \psi_{2}\right)=\left(\begin{array}{lll}
\left\langle\psi_{1}, \partial_{1} H(\mathbf{u}) \psi_{2}\right\rangle & \left\langle\psi_{1}, \partial_{1} H(\mathbf{u}) \psi_{2}\right\rangle^{*} & \left\langle\psi_{2}, \partial_{1} H(\mathbf{u}) \psi_{2}\right\rangle-\left\langle\psi_{1}, \partial_{1} H(\mathbf{u}) \psi_{1}\right\rangle \\
\left\langle\psi_{1}, \partial_{2} H(\mathbf{u}) \psi_{2}\right\rangle & \left\langle\psi_{1}, \partial_{2} H(\mathbf{u}) \psi_{2}\right\rangle^{*} & \left\langle\psi_{2}, \partial_{2} H(\mathbf{u}) \psi_{2}\right\rangle-\left\langle\psi_{1}, \partial_{2} H(\mathbf{u}) \psi_{1}\right\rangle \\
\left\langle\psi_{1}, \partial_{3} H(\mathbf{u}) \psi_{2}\right\rangle & \left\langle\psi_{1}, \partial_{3} H(\mathbf{u}) \psi_{2}\right\rangle^{*} & \left\langle\psi_{2}, \partial_{3} H(\mathbf{u}) \psi_{2}\right\rangle-\left\langle\psi_{1}, \partial_{3} H(\mathbf{u}) \psi_{1}\right\rangle
\end{array}\right) .
$$

Remark IV.2 By simple computations it is easy to see that for every $\mathbf{u}$ and every orthonormal pair $\psi_{1}, \psi_{2} \in \mathcal{D}(H(0))$, $\operatorname{det} \mathcal{M}_{\mathbf{u}}\left(\psi_{1}, \psi_{2}\right)$ is purely imaginary and the function $\left(\psi_{1}, \psi_{2}\right) \mapsto \operatorname{det} \mathcal{M}_{\mathbf{u}}\left(\psi_{1}, \psi_{2}\right)$ is invariant under unitary transformations of the argument.

The following result characterizes conical intersections in terms of the conicity matrix.

Proposition IV.3 Let $H: \mathbb{R}^{3} \rightarrow \mathcal{B}_{s a}(\mathcal{D}(H(0)), \mathcal{H})$ be a $\mathcal{C}^{1}$ map such that $H(\mathbf{u})-H(0)$ is $H(0)$-small for every $\mathbf{u}$. Assume that $\lambda_{1}$ and $\lambda_{2}$ are two discrete eigenvalues of $H(\mathbf{u})$ with $\lambda_{1}(\overline{\mathbf{u}})=\lambda_{2}(\overline{\mathbf{u}})$, and that $\lambda_{1}(\overline{\mathbf{u}})=\lambda_{2}(\overline{\mathbf{u}})$ is a double eigenvalue of $H(\overline{\mathbf{u}})$. Let $\left\{\psi_{1}, \psi_{2}\right\}$ be an orthonormal basis of the eigenspace associated with the double eigenvalue. Then $\overline{\mathbf{u}}$ is a conical intersection if and only if $\mathcal{M}_{\overline{\mathbf{u}}}\left(\psi_{1}, \psi_{2}\right)$ is nonsingular.

Proof. Taking advantage of Proposition A.8, one can easily adapt the proof of Ref. 9, Proposition 3.4 to prove that, if $\overline{\mathbf{u}}$ is a conical intersection, then $\mathcal{M}_{\overline{\mathbf{u}}}\left(\psi_{1}, \psi_{2}\right)$ is nonsingular.

Let us then prove the converse implication. By contradiction, we assume that $\overline{\mathbf{u}}$ is not conical. Let $I$ be a small interval such that $\sigma(H(\overline{\mathbf{u}})) \cap I=\left\{\lambda_{1}(\overline{\mathbf{u}})\right\}$ and denote with $P_{I}(\mathbf{u})$ the spectral projection on $I$ of $H(\mathbf{u})$, i.e., the orthogonal projection onto the sum of the eigenspaces of $H(\mathbf{u})$ associated with the eigenvalues in $I$. For $\mathbf{u}$ belonging to a sufficiently small neighborhood of $\overline{\mathbf{u}}$, we consider the self-adjoint operator $\widetilde{H}(\mathbf{u})=S(\mathbf{u})^{-1} H(\mathbf{u}) S(\mathbf{u})$, 
where $S(\mathbf{u}): P_{I}(\overline{\mathbf{u}}) \mathcal{H} \rightarrow P_{I}(\mathbf{u}) \mathcal{H}$ is the map from the eigenspace relative to $\lambda_{1}(\overline{\mathbf{u}})$ to the range of $P_{I}(\mathbf{u})$ defined as

$$
S(\mathbf{u})=\left.P_{I}(\mathbf{u})\left(\mathrm{id}+P_{I}(\overline{\mathbf{u}})\left(P_{I}(\mathbf{u})-P_{I}(\overline{\mathbf{u}})\right) P_{I}(\overline{\mathbf{u}})\right)^{-1 / 2}\right|_{P_{I}(\overline{\mathbf{u}}) \mathcal{H}} \cdot
$$

It is possible to prove that $S(\mathbf{u})$ is an isometric transformation continuously differentiable with respect to $\mathbf{u}$ (see e.g. Ref. 30, Section 105). $\widetilde{H}(\mathbf{u})$ is represented by a $\mathcal{C}^{1}$ Hermitian 2-dimensional matrix, with eigenvalues $\lambda_{1}(\mathbf{u})$ and $\lambda_{2}(\mathbf{u})$; moreover, $\left\{\widetilde{\psi}_{1}, \widetilde{\psi}_{2}\right\}$, with $\widetilde{\psi}_{i}=$ $S(\overline{\mathbf{u}})^{-1} \psi_{i}$, form an orthonormal basis of the eigenspace of $\widetilde{H}(\overline{\mathbf{u}})$ relative to $\lambda_{1}(\overline{\mathbf{u}})=\lambda_{2}(\overline{\mathbf{u}})$. In particular, the conicity matrix for $\widetilde{H}(\cdot)$ at $\overline{\mathbf{u}}$ with respect to $\left\{\widetilde{\psi}_{1}, \widetilde{\psi}_{2}\right\}$ coincides with that of $H(\cdot)$ at $\overline{\mathbf{u}}$ with respect to $\left\{\psi_{1}, \psi_{2}\right\}$. Indeed,

$$
\begin{aligned}
\partial_{i} \widetilde{H}(\mathbf{u}) & =-S(\mathbf{u})^{-1} \partial_{i} S(\mathbf{u}) S(\mathbf{u})^{-1} H(\mathbf{u}) S(\mathbf{u})+S(\mathbf{u})^{-1} \partial_{i} H(\mathbf{u}) S(\mathbf{u})+S(\mathbf{u})^{-1} H(\mathbf{u}) \partial_{i} S(\mathbf{u}) \\
& =-S(\mathbf{u})^{-1} \partial_{i} S(\mathbf{u}) \widetilde{H}(\mathbf{u})+S(\mathbf{u})^{-1} \partial_{i} H(\mathbf{u}) S(\mathbf{u})+\widetilde{H}(\mathbf{u}) S(\mathbf{u})^{-1} \partial_{i} S(\mathbf{u}),
\end{aligned}
$$

so that $\partial_{i} \widetilde{H}(\overline{\mathbf{u}})=S(\overline{\mathbf{u}})^{-1} \partial_{i} H(\overline{\mathbf{u}}) S(\overline{\mathbf{u}})$, since $\widetilde{H}(\overline{\mathbf{u}})=\lambda_{1}(\overline{\mathbf{u}})$ id.

Without loss of generality, we assume $\widetilde{H}(\mathbf{u})$ traceless for every $\mathbf{u}$. Indeed, the transformation $\widetilde{H}(\mathbf{u}) \mapsto \widetilde{H}(\mathbf{u})-\frac{1}{2} \operatorname{tr}(\widetilde{H}(\mathbf{u}))$ id preserves the difference between the eigenvalues and leads to the same conicity matrix.

Set, for every unit vector $\mathbf{v}$ in $\mathbb{R}^{3}, r_{\mathbf{v}}(t)=\overline{\mathbf{u}}+t \mathbf{v}$. Since $\overline{\mathbf{u}}$ is not conical, for every $\varepsilon>0$ there exists a unit vector $\mathbf{v}_{\varepsilon}=\left(v_{1}^{\varepsilon}, v_{2}^{\varepsilon}, v_{3}^{\varepsilon}\right)$ such that

$$
\left|\frac{d}{d t} \lambda_{1}\left(r_{\mathbf{v}_{\varepsilon}}(t)\right)_{t=0^{+}}\right| \leq \varepsilon
$$

Also, the absolute value of each matrix element of $\widetilde{H}(\mathbf{u})$ is bounded by $\left|\lambda_{1}(\mathbf{u})\right|$, and by continuous differentiability of both $\widetilde{H}$ and $\lambda_{1}$ along $r_{\mathbf{v}_{\varepsilon}}(\cdot)$ (Proposition A.7), one deduces that, in the usual matrix norm induced by the Euclidean norm, $\left\|\frac{d}{d t} \widetilde{H}\left(r_{\mathbf{v}_{\varepsilon}}(t)\right)_{\left.\right|_{t=0^{+}}}\right\|=\left\|\nabla \widetilde{H}(\overline{\mathbf{u}}) \cdot \mathbf{v}_{\epsilon}\right\| \leq$ $C \epsilon$, for some positive $C$.

We now multiply on the left the conicity matrix of $\widetilde{H}(\cdot)$ at $\overline{\mathbf{u}}$ by an orthonormal matrix having $\mathbf{v}_{\epsilon}$ as first row; the resulting matrix has the same determinant as the original one. In particular, the first row of the resulting matrix is

$$
\left(\left\langle\psi_{1}, \nabla \widetilde{H}(\overline{\mathbf{u}}) \cdot \mathbf{v}_{\epsilon} \psi_{2}\right\rangle,\left\langle\psi_{1}, \nabla \widetilde{H}(\overline{\mathbf{u}}) \cdot \mathbf{v}_{\epsilon} \psi_{2}\right\rangle^{*},\left\langle\psi_{2}, \nabla \widetilde{H}(\overline{\mathbf{u}}) \cdot \mathbf{v}_{\epsilon} \psi_{2}\right\rangle-\left\langle\psi_{1}, \nabla \widetilde{H}(\overline{\mathbf{u}}) \cdot \mathbf{v}_{\epsilon} \psi_{1}\right\rangle\right),
$$

so that, by the estimates done above and the arbitrariness of $\epsilon$, we get the proof.

The following result shows a robustness property of conical intersections. The proof may be obtained by following the same arguments as in Ref. 9, Theorem 4.10 and is thus omitted. 
Theorem IV.4 Let $H: \mathbb{R}^{3} \rightarrow \mathcal{B}_{s a}(\mathcal{D}(H(0)), \mathcal{H})$ be a $\mathcal{C}^{1}$ map such that $H(\mathbf{u})-H(0)$ is $H(0)$-small for every $\mathbf{u}$, and let $\overline{\mathbf{u}}$ be a conical intersection for $H(\cdot)$ between the eigenvalues $\lambda_{1}$ and $\lambda_{2}$. Let $U$ be an open neighborhood of $\overline{\mathbf{u}}$. Then, for every $\varepsilon>0$ there exists $\delta>0$ such that, for every $\hat{H}(\cdot)$ satisfying the same assumptions of $H(\cdot)$ and such that

$$
\begin{aligned}
\|H(\mathbf{u})-\hat{H}(\mathbf{u})\|_{H(0)} & \leq \delta \\
\left\|\partial_{i} H(\mathbf{u})-\partial_{i} \hat{H}(\mathbf{u})\right\|_{H(0)} & \leq \delta, \quad i=1,2,3,
\end{aligned}
$$

for $\mathbf{u} \in U$, there exists $\hat{\mathbf{u}} \in \mathbb{R}^{3}$, with $|\overline{\mathbf{u}}-\hat{\mathbf{u}}| \leq \varepsilon$, that is a conical intersection of $\hat{H}(\cdot)$ between $\lambda_{1}$ and $\lambda_{2}$.

\section{MAIN RESULTS}

In the following, we consider a $\mathcal{C}^{1}$ map $K$ defined from a Banach space $\mathcal{Y}$ to $\mathcal{B}_{s a}(\mathcal{D}(K(0)), \mathcal{H})$, such that $K(q)-K(0)$ is $K(0)$-small for every $q \in \mathcal{Y}$. We are interested in establishing the genericity and prevalence of conical intersections for parameter-dependent self-adjoint operators belonging to the class

$$
\mathcal{F}=\{K(q): q \in \mathcal{Y}\}
$$

We also assume that the family $\mathcal{F}$ satisfies the following condition, called Second Strong Arnold Hypothesis ${ }^{10,32}$.

Second Strong Arnold Hypothesis (SAH2): Assume that, for some $q_{0} \in \mathcal{Y}, \lambda$ is an eigenvalue of $K\left(q_{0}\right)$ of multiplicity greater or equal than two. Then there exist two orthonormal eigenstates $\psi_{1}, \psi_{2}$ of $K\left(q_{0}\right)$ pertaining to $\lambda$ such that the three linear functionals on $\mathcal{Y}$

$$
\begin{aligned}
f_{11}-f_{22}: p & \mapsto\left\langle\psi_{1}, K_{q_{0}}^{\prime}(p) \psi_{1}\right\rangle-\left\langle\psi_{2}, K_{q_{0}}^{\prime}(p) \psi_{2}\right\rangle \\
f_{12}: p & \mapsto\left\langle\psi_{1}, K_{q_{0}}^{\prime}(p) \psi_{2}\right\rangle \\
f_{21}: p & \mapsto\left\langle\psi_{2}, K_{q_{0}}^{\prime}(p) \psi_{1}\right\rangle
\end{aligned}
$$

are linearly independent. Equivalently, the linear map $\Phi=\left(f_{11}-f_{22}, \mathfrak{R e}\left(f_{12}\right), \mathfrak{I m}\left(f_{12}\right)\right)$ is surjective from $\mathcal{Y}$ to $\mathbb{R}^{3}$.

We call $\mathcal{M}$ the subset of $\mathcal{Y}$ such that the operators in $K(\mathcal{M})$ have double eigenvalues. For every interval $I$ and every open $\operatorname{set} \mathcal{U}$ in $\mathcal{Y}$, we denote by $\mathcal{M}^{I, \mathcal{U}}$ the subset of elements 
in $\mathcal{U}$ such that the corresponding operators have an eigenvalue in $I$ of multiplicity two, isolated from the rest of the spectrum. Under some additional regularity assumptions on the spectrum of the operators, SAH2 guarantees that $\mathcal{M}$ has codimension three in $\mathcal{Y}$. In particular if, for any $q \in \mathcal{Y}, K(q)$ only admits point spectrum with no finite accumulation points, then, for a sufficiently small interval $I$ and a sufficiently small open set $\mathcal{U}$, the set $\mathcal{M}^{I, \mathcal{U}}$ is a smooth submanifold of codimension three ${ }^{32}$.

The conicity of eigenvalues intersections corresponds to a geometric property in the space of parameters, as the following result shows.

Proposition V.1 Assume that the family $\mathcal{F}$ satisfies $\mathbf{S A H 2}$, and that all of its elements have purely discrete spectrum without finite accumulation points. Consider a $\mathcal{C}^{1}$ map $q$ : $U \rightarrow \mathcal{Y}$, with $U \subset \mathbb{R}^{3}$ open, and set $H(\mathbf{u})=K(q(\mathbf{u}))$. Assume that $H(\mathbf{u})$ has an isolated double eigenvalue $\lambda$ at $\mathbf{u}=\overline{\mathbf{u}}$, and set $\bar{q}=q(\overline{\mathbf{u}})$. Consider a neighborhood $\mathcal{U}$ of $\bar{q}$ in $\mathcal{Y}$ and an interval I containing $\lambda$, small enough so that $\mathcal{M}^{I, \mathcal{U}}$ is a submanifold of codimension three in $\mathcal{Y}$. Then $\overline{\mathbf{u}}$ is a conical intersection for $H(\cdot)$ if and only if for every direction $\mathbf{v} \in \mathbb{R}^{3}$ the vector $q_{\mathbf{v}}=\sum_{i=1}^{3} v_{i} \partial_{i} q(\overline{\mathbf{u}})$ is not tangent to $\mathcal{M}^{I, \mathcal{U}}$ at $\bar{q}$, that is, the map $q(\cdot)$ is transversal to $\mathcal{M}^{I, \mathcal{U}}$ at $\overline{\mathbf{u}}$.

Proof. If there exists some $\mathbf{v} \in \mathbb{R}^{3}$ such that $q_{\mathbf{v}}$ is tangent to $\mathcal{M}^{I, \mathcal{U}}$ at $\bar{q}$, then $\overline{\mathbf{u}}$ cannot be a conical intersection. Indeed, if a curve $\gamma$ in $\mathcal{M}^{I, \mathcal{U}}$ satisfies $\gamma(0)=\overline{\mathbf{u}}$ and $\dot{\gamma}(0)=q_{\mathbf{v}}$, then

$$
\|K(\gamma(t))-K(q(\overline{\mathbf{u}}+t \mathbf{v}))\|_{\mathcal{D}(K(0))}=o(t) .
$$

Thanks to Proposition A.6, we get that the distance between the eigenvalues intersecting at $\overline{\mathbf{u}}$ is of order $o(t)$ along the line $t \mapsto \overline{\mathbf{u}}+t \mathbf{v}$.

Let us now prove the converse statement. Denote by $\lambda_{1}(\mathbf{u})$ and $\lambda_{2}(\mathbf{u})$ the two eigenvalues of $H(\mathbf{u})$ crossing at $\overline{\mathbf{u}}$, with $\lambda_{1}(\overline{\mathbf{u}})=\lambda_{2}(\overline{\mathbf{u}})=\lambda$.

Under the assumptions of the proposition, we can deduce the following facts. Possibly reducing $I$ (still containing $\lambda$ in its interior) and the neighborhood $\mathcal{U}$ of $\bar{q}, K(q)$ contains exactly two eigenvalues in $I$, counted with their multiplicity, for every $q \in \mathcal{U}$. Denoting with $M(q)$ the sum of the eigenspaces of $K(q)$ associated with the eigenvalues in $I$, and with $P_{I}(q)$ the orthogonal projection on $M(q)$, we have that, possibly reducing $\mathcal{U}$,

$$
\left\|P_{I}(q)-\bar{P}\right\|<1 \quad \forall q \in \mathcal{U}
$$


where $\bar{P}=P_{I}(\bar{q})$; moreover, $P_{I}(\cdot)$ is $\mathcal{C}^{1}$ in $\mathcal{U}$, by Proposition A.4.

Define the map $S(q): M(\bar{q}) \rightarrow M(q)$

$$
S(q)=\left.P_{I}(q)\left(\mathrm{id}+\bar{P}\left(P_{I}(q)-\bar{P}\right) \bar{P}\right)^{-1 / 2}\right|_{M(\bar{q})} .
$$

As in the proof of Proposition IV.3, we can see that $S(q)$ is an isometric transformation and it is differentiable with respect to $q$. Therefore the map

$$
h(q)=S(q)^{-1} K(q) S(q)
$$

is a differentiable map from $\mathcal{U}$ to the space of self-adjoint operators on $M(\bar{q})$, and the eigenvalues of $h(q)$ are the same as the eigenvalues of $K(q)$ in $I$. Moreover, it is easy to see that $\mathcal{M}^{I, \mathcal{U}} \subset h^{-1}(\{\mu$ id $: \mu \in \mathbb{R}\})$, where id denotes the identity on $M(\bar{q})$.

Let us now assume that the intersection between the eigenvalues $\lambda_{1}$ and $\lambda_{2}$ is not conical, that is there exists a unit vector $\mathbf{v} \in \mathbb{R}^{3}$ such that

$$
\lambda_{2}(\overline{\mathbf{u}}+t \mathbf{v})-\lambda_{1}(\overline{\mathbf{u}}+t \mathbf{v})=o(t) .
$$

We claim that $q_{\mathbf{v}}$ is tangent to $\mathcal{M}^{I, \mathcal{U}}$ at $\bar{q}$. To prove that, we consider the curve $h(q(\overline{\mathbf{u}}+t \mathbf{v}))$ in the space of self-adjoint operators on $M(\bar{q})$. Choosing $\left\{\psi_{1}, \psi_{2}\right\}$ as the orthonormal basis of $M(\bar{q})$ for which SAH2 holds true, we represent $h(q(\overline{\mathbf{u}}+t \mathbf{v}))$ as the curve of two dimensional Hermitian matrices

$$
N(t)=\left(\begin{array}{ll}
a(t) & c(t) \\
c^{*}(t) & b(t)
\end{array}\right),
$$

for some complex-valued functions $a(\cdot), b(\cdot), c(\cdot)$ satisfying $a(0)=b(0)=\lambda$ and $c(0)=0$. Since the eigenvalues of $N(t)$ coincide with those of $H(\overline{\mathbf{u}}+t \mathbf{v})$ contained in $I$, it holds $\sqrt{(a(t)-b(t))^{2}+4|c(t)|^{2}}=o(t)$. Therefore, we conclude that $\dot{N}(0)$ belongs to $\{\mu$ id $: \mu \in$ $\mathbb{R}\}$.

Reasoning as in Proposition IV.3, we obtain that $h_{\bar{q}}^{\prime}(\cdot)=S^{-1}(\bar{q}) K_{\bar{q}}^{\prime}(\cdot) S(\bar{q})$. Since the family $\mathcal{F}$ satisfies the condition SAH2, the map $h$ is transversal to $\{\mu$ id $: \mu \in \mathbb{R}\}$ in the space of self-adjoint operators on $M(\bar{q})$. Then we can conclude that

$$
\left(h_{\bar{q}}^{\prime}\right)^{-1}(\{\mu \mathrm{id}: \mu \in \mathbb{R}\})=T_{\bar{q}} \mathcal{M}^{I, \mathcal{U}}
$$

(see e.g. Ref. 1, Corollary 17.2) and, in particular, $q_{\mathbf{v}}$ is tangent to $\mathcal{M}^{I, \mathcal{U}}$ at $\bar{q}$.

Note that, if $\mathcal{Y}$ is second countable and if all the elements of $\mathcal{F}$ have purely discrete spectrum without finite accumulation points, then the set $\mathcal{M}$ is the countable union of 
manifolds of the form $\mathcal{M}^{I, \mathcal{U}}$, as defined above. Hence, from the previous result, the fact that the family of prevalent and residual sets is closed under countable intersection, and by Theorem III.4, we deduce the following criterion discussing the possibility, for an element of a family of parameter-dependent self-adjoint operators, that all double eigenvalues intersections are conical.

Theorem V.2 Assume that the family $\mathcal{F}$ satisfies $\mathbf{S A H 2}$, and that all of its elements have purely discrete spectrum without finite accumulation points. Let $\mathcal{A}$ be a Banach space, $U \subset \mathbb{R}^{3}$ open and ev $: \mathcal{A} \times U \rightarrow \mathcal{Y}$ be a $\mathcal{C}^{1}$ map. Furthermore, suppose that $\mathcal{A}, \mathcal{Y}$ are second countable and that the map $\mathrm{ev}(\cdot)$ is transverse to the manifolds of double eigenvalues $\mathcal{M}^{I, \mathcal{U}}$ defined above. Then, for a residual and prevalent set of elements a $\in \mathcal{A}$, all double eigenvalues intersections of the parameter-dependent operator $H_{a}(\cdot)=K(\operatorname{ev}(a, \cdot))$ are conical.

\section{A. Finite-dimensional case}

In this section, we consider finite dimensional parameterized Hamiltonians of the form $H(\mathbf{u})=H_{0}+u_{1} H_{1}+u_{2} H_{2}+u_{3} H_{3}$ where, for $i=0, \ldots, 3, H_{i}$ is a $n$-dimensional Hermitian matrix. In this context, genericity and prevalence have to be intended with respect to the Hamiltonians $H_{i}, \quad i=1,2,3$.

In the notations of the preceding section, we have that $\mathcal{F}=i \mathfrak{u}(n)$, that is, $\mathcal{F}$ is the set of Hermitian $n \times n$ matrices, and we choose $\mathcal{Y}=\mathcal{F}$ and $K$ the identity on $\mathcal{Y}$. All the conditions on $\mathcal{F}, \mathcal{A}, \mathcal{Y}$ in Theorem V.2 are satisfied, and in particular, for sufficiently small $I \subset \mathbb{R}$ and $\mathcal{U} \subset \mathcal{Y}$, the set $\mathcal{M}^{I, \mathcal{U}}$ of double eigenvalues is a submanifold of codimension 3 in $i \mathfrak{u}(n)$.

Lemma V.3 Fix $H_{0} \in i \mathfrak{u}(n)$. We define the map ev $:(i \mathfrak{u}(n))^{3} \times\left(\mathbb{R}^{3} \backslash\{0\}\right) \rightarrow i \mathfrak{u}(n)$ as

$$
\mathrm{ev}\left(H_{1}, H_{2}, H_{3}, \mathbf{u}\right)=H(\mathbf{u})
$$

with $H(\mathbf{u})=H_{0}+u_{1} H_{1}+u_{2} H_{2}+u_{3} H_{3}$. Then ev is transversal to the manifolds $\mathcal{M}^{I, \mathcal{U}}$.

Proof. If $\mathrm{ev}\left(H_{1}, H_{2}, H_{3}, \mathbf{u}\right) \notin \mathcal{M}^{I, \mathcal{U}}$, then the thesis trivially holds. Assume then that $H(\overline{\mathbf{u}})=\mathrm{ev}\left(H_{1}, H_{2}, H_{3}, \overline{\mathbf{u}}\right) \in \mathcal{U}$ has a double eigenvalue $\lambda \in I$ for $\overline{\mathbf{u}} \neq 0$. 
The differential of ev at $\left(H_{1}, H_{2}, H_{3}, \overline{\mathbf{u}}\right)$ along the direction $\left(\delta H_{1}, \delta H_{2}, \delta H_{3}, \delta \mathbf{u}\right)$ is given by

$$
\mathrm{ev}_{\left(H_{1}, H_{2}, H_{3}, \overline{\mathbf{u}}\right)}^{\prime}\left(\delta H_{1}, \delta H_{2}, \delta H_{3}, \delta \mathbf{u}\right)=\sum_{j=1}^{3}\left(\bar{u}_{j} \delta H_{j}+\delta u_{j} H_{j}\right) .
$$

Let us consider the three directions $v_{l}=\left(\bar{u}_{1} \sigma_{l}, \bar{u}_{2} \sigma_{l}, \bar{u}_{3} \sigma_{l}, 0,0,0\right) \in(i \mathfrak{u}(n))^{3} \times \mathbb{R}^{3}$, where we introduce Pauli-like operators $\sigma_{l}, l=1,2,3$ as follows

$$
\sigma_{1}=\left\langle\varphi_{2}, \cdot\right\rangle \varphi_{1}+\left\langle\varphi_{1}, \cdot\right\rangle \varphi_{2} \quad \sigma_{2}=i\left\langle\varphi_{1}, \cdot\right\rangle \varphi_{2}-i\left\langle\varphi_{2}, \cdot\right\rangle \varphi_{1} \quad \sigma_{3}=\left\langle\varphi_{1}, \cdot\right\rangle \varphi_{1}-\left\langle\varphi_{2}, \cdot\right\rangle \varphi_{2}
$$

and $\varphi_{1}$ and $\varphi_{2}$ define an orthonormal basis of the eigenspace of $H(\overline{\mathbf{u}})$ relative to $\lambda$. We now consider the eigenvalues of

$$
\begin{aligned}
H(\overline{\mathbf{u}}) & +\epsilon \operatorname{ev}_{\left(H_{1}, H_{2}, H_{3}, \overline{\mathbf{u}}\right)}^{\prime}\left(\alpha_{1} v_{1}+\alpha_{2} v_{2}+\alpha_{3} v_{3}\right) \\
& =H(\overline{\mathbf{u}})+\epsilon|\overline{\mathbf{u}}|^{2} \sum_{j=1}^{3} \alpha_{j} \sigma_{j} .
\end{aligned}
$$

It is easy to check that the degenerate eigenvalues split and their difference is equal to $2 \epsilon|\overline{\mathbf{u}}|^{2}|\alpha|$. Therefore, $\operatorname{span}\left\{\operatorname{ev}_{\left(H_{1}, H_{2}, H_{3}, \overline{\mathbf{u}}\right)}^{\prime}\left(v_{l}\right): l=1,2,3\right\}$ is a three dimensional space having trivial intersection with $T_{H(\overline{\mathbf{u}})} \mathcal{M}^{I, \mathcal{U}}$. Since the codimension of $\mathcal{M}^{I, \mathcal{U}}$ is 3 , we conclude that the map ev is transversal to $\mathcal{M}^{I, \mathcal{U}}$.

We are now able to state the main result of this section.

Theorem V.4 Let $H_{0} \in i \mathfrak{u}(n)$. With each $\left(H_{1}, H_{2}, H_{3}\right) \in(i \mathfrak{u}(n))^{3}$, we associate the parameter-dependent operator $H(\mathbf{u})=H_{0}+\sum_{j=1}^{3} u_{j} H_{j}$. Then the set of triples $\left(H_{1}, H_{2}, H_{3}\right)$ such that all double eigenvalues of $H(\cdot)$ correspond to conical intersections is residual and of full Lebesgue measure.

Proof. We consider separately the two cases in which $H_{0}$ admits or does not admit double eigenvalues.

Thanks to Lemma V.3 we can apply Theorem V.2 with $U=\mathbb{R}^{3} \backslash\{0\}$ and deduce that, for a residual and prevalent (hence full measure) subset of $(i \mathfrak{u}(n))^{3}$, all double eigenvalues of $H(\mathbf{u})$ with $\mathbf{u} \neq 0$ correspond to conical intersections. This is enough to conclude the proof in the case where $H_{0}$ has no double eigenvalues.

Let us now consider the case where $H_{0}$ has a double eigenvalue $\lambda$, and let $\psi_{1}$ and $\psi_{2}$ define an orthonormal basis of the eigenspace relative to $\lambda$. Consider the real-valued multi-linear 
map

$$
F\left(H_{1}, H_{2}, H_{3}\right)=\operatorname{det}\left(\begin{array}{lll}
\mathfrak{R e}\left(\left\langle\psi_{1}, H_{1} \psi_{2}\right\rangle\right) & \mathfrak{I m}\left(\left\langle\psi_{1}, H_{1} \psi_{2}\right\rangle\right) & \left(\left\langle\psi_{2}, H_{1} \psi_{2}\right\rangle-\left\langle\psi_{1}, H_{1} \psi_{1}\right\rangle\right) \\
\mathfrak{R e}\left(\left\langle\psi_{1}, H_{2} \psi_{2}\right\rangle\right) & \mathfrak{I m}\left(\left\langle\psi_{1}, H_{2} \psi_{2}\right\rangle\right) & \left(\left\langle\psi_{2}, H_{2} \psi_{2}\right\rangle-\left\langle\psi_{1}, H_{2} \psi_{1}\right\rangle\right) \\
\mathfrak{R e}\left(\left\langle\psi_{1}, H_{3} \psi_{2}\right\rangle\right) & \mathfrak{I m}\left(\left\langle\psi_{1}, H_{3} \psi_{2}\right\rangle\right) & \left(\left\langle\psi_{2}, H_{3} \psi_{2}\right\rangle-\left\langle\psi_{1}, H_{3} \psi_{1}\right\rangle\right)
\end{array}\right) .
$$

By Proposition IV.3, $\mathbf{u}=0$ is a conical intersection for $H_{0}+\sum_{j=1}^{3} u_{j} H_{j}$ if and only if $F\left(H_{1}, H_{2}, H_{3}\right) \neq 0$. Moreover, being $F$ continuous, we obtain that $F^{-1}(\mathbb{R} \backslash\{0\})$ is an open subset of $(i \mathfrak{u}(n))^{3}$. This subset is nonempty because the map

$$
H \in i \mathfrak{u}(n) \mapsto\left(\mathfrak{R e}\left(\left\langle\psi_{1}, H \psi_{2}\right\rangle\right), \mathfrak{I m}\left(\left\langle\psi_{1}, H \psi_{2}\right\rangle\right),\left\langle\psi_{2}, H \psi_{2}\right\rangle-\left\langle\psi_{1}, H \psi_{1}\right\rangle\right) \in \mathbb{R}^{3}
$$

is surjective. The density comes directly from multi-linearity. Also, the set $F^{-1}(\mathbb{R} \backslash\{0\})$ has full measure in $(i \mathfrak{u}(n))^{3}$. Since $H_{0}$ possesses at most countably many double eigenvalues, the set of triples $\left(H_{1}, H_{2}, H_{2}\right) \in(i \mathfrak{u}(n))^{3}$ for which all double eigenvalues intersections at the origin are conical is then the intersection of residual and full measure subsets of $(i \mathfrak{u}(n))^{3}$. Further intersecting with the subset of $(i \mathfrak{u}(n))^{3}$ provided in the first part of the proof, we obtain a residual and full measure set of matrices $\left(H_{1}, H_{2}, H_{2}\right) \in(i \mathfrak{u}(n))^{3}$ such that all double eigenvalues of $H(\mathbf{u})$ with $\mathbf{u} \in \mathbb{R}^{3}$ correspond to conical intersections. This concludes the proof of the theorem.

\section{B. Infinite dimension: the case of electromagnetic Hamiltonians}

In quantum mechanics, infinite dimensional Hamiltonians are usually obtained by quantization of classical Hamiltonians, that is, sums of kinetic and potential energy. In particular, a charged particle moving in the three dimensional space under the action of an electromagnetic field (E, B) is described by the following Hamiltonian

$$
\begin{aligned}
H & =(-i \nabla+\mathbf{A})^{2}+V \\
& =(-i \nabla+\mathbf{A}) \cdot(-i \nabla+\mathbf{A})+V \\
& =-\Delta-i(\nabla \mathbf{A}+\mathbf{A} \nabla)+|\mathbf{A}|^{2}+V
\end{aligned}
$$

where $\mathbf{A}: \mathbb{R}^{3} \rightarrow \mathbb{R}^{3}$ is the vector potential and $V: \mathbb{R}^{3} \rightarrow \mathbb{R}$ is the scalar potential, and they satisfy the relations $\mathbf{E}=-\nabla V$ and $\mathbf{B}=\nabla \times \mathbf{A}$. Here the operator $-i(\nabla \mathbf{A}+\mathbf{A} \nabla)$ acts on the elements of its domain as follows:

$$
-i(\nabla \mathbf{A}+\mathbf{A} \nabla) \psi=-i \operatorname{div}(\mathbf{A} \psi)-i \mathbf{A} \cdot \nabla \psi
$$


The class of Hamiltonians of the form (2) covers many relevant physical systems, therefore it is a good candidate for the class $\mathcal{F}$ in which to study the occurrence of conical intersections. Notice that the Hamiltonian (2) can be written as $H=-\Delta+W+H_{\mathbf{A}}$, where we set $H_{\mathbf{A}}=$ $-i(\nabla \mathbf{A}+\mathbf{A} \nabla)$ and $W=V+|\mathbf{A}|^{2}$. Formally, $\mathcal{H}=L^{2}(\Omega, \mathbb{C})$ where $\Omega$ is a given Lipschitz bounded domain in $\mathbb{R}^{3}$, the class $\mathcal{F}$ under consideration consists of the Hamiltonians of the form $-\Delta+W+H_{\mathbf{A}}$, where $\Delta: \mathcal{D}(\Delta) \rightarrow L^{2}(\Omega, \mathbb{C})$ denotes the Dirichlet Laplacian on $\Omega$ with $\mathcal{D}(\Delta)=H_{0}^{1}(\Omega, \mathbb{C}) \cap H^{2}(\Omega, \mathbb{C}), W$ is a scalar continuous real-valued function on its closure $\bar{\Omega}$, that should be thought as a multiplication operator, and $\mathbf{A}$ is a $\mathcal{C}^{1}$ vector-valued real function from $\bar{\Omega}$ to $\mathbb{R}^{3}$. Using the fact that $\|\nabla \psi\|=|\langle\psi, \Delta \psi\rangle|^{1 / 2} \leq \frac{1}{2}\left(\varepsilon\|\Delta \psi\|+\frac{1}{\varepsilon}\|\psi\|\right)$ for any $\varepsilon>0$, one gets

$$
\left\|H_{\mathbf{A}} \psi\right\|=\|\operatorname{div}(\mathbf{A}) \psi+2 \mathbf{A} \cdot \nabla \psi\| \leq \varepsilon\|\mathbf{A}\|_{L^{\infty}(\Omega)}\|\Delta \psi\|+\left(\|\operatorname{div}(\mathbf{A})\|_{L^{\infty}(\Omega)}+\frac{1}{\varepsilon}\|\mathbf{A}\|_{L^{\infty}(\Omega)}\right)\|\psi\| .
$$

As a consequence, self-adjoint operators of the form $H_{\mathbf{A}}$ are $\Delta$-small. In particular the elements of $\mathcal{F}$ belong to $\mathcal{B}_{\text {sa }}\left(\mathcal{D}(\Delta), L^{2}(\Omega)\right.$ ). Similarly it can be shown that each $H_{\mathbf{A}}$ is formbounded with respect to $-\Delta$ (as a quadratic form, see Ref. 27 , Chapter X for the definition) with a relative bound that can be chosen smaller than one. Thus Ref. 28, Theorem XIII.68 ensures that the Hamiltonians of the form $-\Delta+V+H_{\mathbf{A}}$ have compact resolvent, so that their spectrum is purely discrete with a finite number of eigenvalues in each compact subset of $\mathbb{R}$.

Before stating the main result of this section, we observe that the operator $H_{\mathbf{A}}$ plays a crucial role in the existence of conical intersections for controlled Hamiltonians belonging to $\mathcal{F}$. Indeed, controlled Hamiltonians of the form $H(\mathbf{u})=-\Delta+V(\mathbf{u}), \mathbf{u} \in \mathbb{R}^{3}$, do not admit conical intersections: this can be seen as a consequence of the fact that the terms $\left\langle\psi_{j}, \partial_{i} H(\mathbf{u}) \psi_{k}\right\rangle$, with $i=1,2,3$, computed with respect to an appropriately chosen orthonormal basis of eigenfunctions $\left\{\psi_{j}(\mathbf{u})\right\}_{j \in \mathbb{N}}$ of $-\Delta+V(\mathbf{u})$, are real and thus the first two columns of each conicity matrix are equal.

On the other hand, examples of conical intersections for controlled Hamiltonians belonging to the family $\mathcal{F}$ defined above are not difficult to find, as shown below. ${ }^{26}$

Example. Consider the Hamiltonian $H(\mathbf{u})=\left(-i \nabla+u_{3} \mathbf{A}\right)^{2}+u_{1} V_{1}+u_{2} V_{2}$, where

$$
\begin{gathered}
V_{1}(x)=x_{2}^{2}+x_{3}^{2}, V_{2}(x)=x_{2} x_{3}, \mathbf{A}=\left(0,-x_{3} / 2, x_{2} / 2\right)^{T}, \\
x=\left(x_{1}, x_{2}, x_{3}\right) \in \Omega=(0,1) \times(0, \sqrt{3}) \times(0, \sqrt{5}),
\end{gathered}
$$


with Dirichlet boundary conditions. We claim that $H(0)$ (representing the potential well in $\Omega$ ) admits conical intersections of eigenvalues. Indeed eigenvalues and eigenfunctions of $H(0)$ take the form

$$
\begin{gathered}
\lambda_{j_{1}, j_{2}, j_{3}}=\pi^{2}\left(j_{1}^{2}+\frac{j_{2}^{2}}{3}+\frac{j_{3}^{2}}{5}\right), \\
\psi_{j_{1}, j_{2}, j_{3}}(x)=\frac{2 \sqrt{2}}{\sqrt[4]{15}} \sin \left(j_{1} \pi x_{1}\right) \sin \left(\frac{j_{2} \pi x_{2}}{\sqrt{3}}\right) \sin \left(\frac{j_{3} \pi x_{3}}{\sqrt{5}}\right),
\end{gathered}
$$

where $j_{1}, j_{2}, j_{3}$ are strictly positive integers. Then, for instance, $\lambda_{1,1,3}=\lambda_{1,2,2}$ corresponds to a double eigenvalue. A direct computation shows that the associated conicity matrix is nonsingular.

We then have the following result.

Theorem V.5 Let $(-i \nabla+\overline{\mathbf{A}})^{2}+\bar{V} \in \mathcal{F}$ and $U=\left\{\mathbf{u} \in \mathbb{R}^{3}: u_{1}^{2}+u_{2}^{2} \neq 0\right.$ and $\left.u_{3} \neq 0\right\}$. Then, for a residual and prevalent set of triples $\left(V_{1}, V_{2}, \mathbf{A}\right)$ in $(\mathcal{C}(\bar{\Omega}, \mathbb{R}))^{2} \times \mathcal{C}^{1}\left(\bar{\Omega}, \mathbb{R}^{3}\right)$, all the double eigenvalues of

$$
H(\mathbf{u})=\left(-i \nabla+\overline{\mathbf{A}}+u_{3} \mathbf{A}\right)^{2}+\bar{V}+u_{1} V_{1}+u_{2} V_{2}
$$

with $\mathbf{u} \in U \cup\{0\}$ correspond to conical intersections.

The proof of Theorem V.5 is based on an application of Theorem V.2. To this end, we set $\mathcal{Y}=\mathcal{C}(\bar{\Omega}, \mathbb{R}) \times \mathcal{C}^{1}\left(\bar{\Omega}, \mathbb{R}^{3}\right)$ and define the surjective map $K: \mathcal{Y} \rightarrow \mathcal{F}$ as

$$
K(V, \mathbf{A})=-\Delta+H_{\mathbf{A}}+|\mathbf{A}|^{2}+V .
$$

We recall that $\mathcal{Y}$, endowed with the norm $\|(V, \mathbf{A})\|_{\mathcal{Y}}=\|V\|_{\infty}+\|\mathbf{A}\|_{\infty}+\sum_{k=1}^{3}\left\|\nabla \mathbf{A}_{k}\right\|_{\infty}$ for $(V, \mathbf{A}) \in \mathcal{Y}$, is a Banach space. Note that, in this context, the assumption that $\Omega$ is bounded is crucial. Indeed, the space of continuous (or $\mathcal{C}^{1}$ ) functions on an unbounded domain of $\mathbb{R}^{3}$ is not second countable. On the other hand, if $\Omega$ is bounded, then $\mathcal{Y}$ is separable and thus second countable (the two notions are equivalent on metric spaces).

It is easy to see that $K$ is differentiable as a map from the Banach space $\mathcal{Y}$ to $\mathcal{B}_{s a}\left(\mathcal{D}(\Delta), L^{2}(\Omega)\right)$, with $K_{(V, \mathbf{A})}^{\prime}(\delta V, \delta \mathbf{A})=H_{\delta \mathbf{A}}+2 \mathbf{A} \cdot \delta \mathbf{A}+\delta V ;$ indeed

$$
\left\|K(V+\delta V, \mathbf{A}+\delta \mathbf{A})-K(V, \mathbf{A})-\left(H_{\delta \mathbf{A}}+2 \mathbf{A} \cdot \delta \mathbf{A}+\delta V\right)\right\|_{\mathcal{B}\left(\mathcal{D}(\Delta), L^{2}(\Omega)\right)}=o\left(\|(\delta V, \delta \mathbf{A})\|_{\mathcal{Y}}\right)
$$

and, because of $(3), H_{\delta \mathbf{A}}+2 \mathbf{A} \cdot \delta \mathbf{A}+\delta V$ is a bounded linear map from $\mathcal{Y}$ to $\mathcal{B}\left(\mathcal{D}(\Delta), L^{2}(\Omega)\right)$. The continuity of $(V, \mathbf{A}) \mapsto K_{(V, \mathbf{A})}^{\prime}$ being evident, we conclude that $K$ is $\mathcal{C}^{1}$. 
In order to apply Theorem V.2 to the family $\mathcal{F}$ and the function $K$ defined above, we will use the following preliminary result.

Lemma V.6 Every element of $\mathcal{F}$ satisfies SAH2 for any multiple eigenvalue. Moreover, the restriction $\left.\Phi\right|_{\{(V, 0): V \in \mathcal{C}(\bar{\Omega}, \mathbb{R})\}}$, where $\Phi$ is the map defined in the statement of $\mathbf{S A H 2}$, has rank at least two.

Proof. Let us consider $\bar{H}=-\Delta+V+|\mathbf{A}|^{2}+H_{\mathbf{A}} \in \mathcal{F}$, and assume that $\lambda$ is a multiple eigenvalue of $\bar{H}$. By contradiction, assume that there exist three complex scalars $a, b, c$ and two eigenstates $\psi_{1}, \psi_{2}$ of $\bar{H}$ relative to the eigenvalue $\lambda$ such that the functional

$$
a\left(f_{11}-f_{22}\right)+b f_{12}+c f_{21}
$$

is identically zero, where $f_{i j}(\delta V, \delta A)=\left\langle\psi_{i},\left(H_{\delta \mathbf{A}}+2 \mathbf{A} \cdot \delta \mathbf{A}+\delta V\right) \psi_{j}\right\rangle$. Notice that this fact does not depend on the particular choice of the orthonormal eigenstates $\psi_{1}, \psi_{2}$. Indeed, a transformation of the basis $\left(\tilde{\psi}_{1}, \tilde{\psi}_{2}\right)^{T}=M\left(\psi_{1}, \psi_{2}\right)^{T}$, where $M$ is an invertible two-by-two matrix, induces a linear invertible transformation $(a, b, c) \mapsto(\tilde{a}, \tilde{b}, \tilde{c})$ so that the functional (4) becomes $\tilde{a}\left(\tilde{f}_{11}-\tilde{f}_{22}\right)+\tilde{b} \tilde{f}_{12}+\tilde{c} \tilde{f}_{21}$, where $\tilde{f}_{i j}=\left\langle\tilde{\psi}_{i},\left(H_{\delta \mathbf{A}}+2 \mathbf{A} \cdot \delta \mathbf{A}+\delta V\right) \tilde{\psi}_{j}\right\rangle$.

Integrating by parts the terms of the kind $\left\langle\psi_{i}, H_{\delta \mathbf{A}} \psi_{j}\right\rangle$ taking into account the boundary conditions on the eigenfunctions, we can write the functional (4) as

$$
(\delta V, \delta \mathbf{A}) \mapsto \int_{\Omega}(\delta V+2 \mathbf{A} \cdot \delta \mathbf{A}) \Theta-i \delta \mathbf{A} \cdot \Xi
$$

where

$$
\begin{aligned}
& \Theta=a\left|\psi_{1}\right|^{2}-a\left|\psi_{2}\right|^{2}+b \psi_{1}^{*} \psi_{2}+c \psi_{1} \psi_{2}^{*}, \\
& \Xi=a\left(\psi_{1}^{*} \nabla \psi_{1}-\psi_{1} \nabla \psi_{1}^{*}-\psi_{2}^{*} \nabla \psi_{2}+\psi_{2} \nabla \psi_{2}^{*}\right)+b\left(\psi_{1}^{*} \nabla \psi_{2}-\psi_{2} \nabla \psi_{1}^{*}\right)+c\left(\psi_{2}^{*} \nabla \psi_{1}-\psi_{1} \nabla \psi_{2}^{*}\right) .
\end{aligned}
$$

Since we are assuming that the functional (5) is zero then, by arbitrariness of $\delta V$ and $\delta \mathbf{A}$, $\Theta$ and $\Xi$ must be identically zero on $\Omega$. We assume without loss of generality that $a \in \mathbb{R}$ and consider separately the cases $b=c^{*}$ and $b \neq c^{*}$.

If $b=c^{*}$ then, up to replacing $\psi_{1}$ with $e^{i \theta} \psi_{1}$ in (4) for a suitable constant phase $\theta \in \mathbb{R}$, we can assume $b=c \in \mathbb{R}$. Furthermore, it is easy to see that a rotation of the basis $\left(\psi_{1}, \psi_{2}\right)^{T} \mapsto R\left(\psi_{1}, \psi_{2}\right)^{T}, R \in S O(2)$, induces a rotation of the corresponding coefficients $a$ and $b=c$ in (4) (more precisely the vector of coefficients $(a, b)^{T}$ is mapped to $\left.R^{2}(a, b)^{T}\right)$. As a consequence we assume without loss of generality that $b=c=0$. From $\Theta=0$ we obtain that 
$\left|\psi_{1}\right| \equiv\left|\psi_{2}\right|$ on $\Omega$. Denoting $\psi_{j}=|\psi| e^{i \theta_{j}}, j=1,2$, it turns out that $\Xi=2 i a|\psi|^{2} \nabla\left(\theta_{1}-\theta_{2}\right)$. Then $\nabla\left(\theta_{1}-\theta_{2}\right)=0$ wherever $|\psi| \neq 0$, and in particular $\theta_{1}-\theta_{2}$ is constant on an open set. Up to a constant phase change of the eigenfunctions, $\psi_{1}-\psi_{2}$ is an eigenfunction which is null on an open set, which implies, by the unique continuation property ${ }^{20}$, that $\psi_{1} \equiv \psi_{2}$, which is a contradiction. We can then conclude that the functional (4) cannot be zero if $a \in \mathbb{R}$ and $b=c^{*}$.

Let now $b \neq c^{*}$. By the unique continuation property, we have that $\psi_{1}$ and $\psi_{2}$ are different from zero on an open and dense subset $\Omega^{\prime} \subset \Omega$. Since $\Theta-\Theta^{*}=\left(b-c^{*}\right) \psi_{1}^{*} \psi_{2}+\left(c-b^{*}\right) \psi_{1} \psi_{2}^{*} \equiv 0$ we then obtain that the difference between the phases of $\psi_{1}$ and $\psi_{2}$ is constant on $\Omega^{\prime}$ and, in particular, it can be set to zero. This leads to $\mathfrak{I m}(b)=-\mathfrak{I m}(c)$. Let us then set $b=\beta+i r$, $c=\gamma-i r$, for some $\beta, \gamma, r \in \mathbb{R}$, with $\beta \neq \gamma$ (otherwise we are back to the previous case). Let us write $\psi_{1}=\phi_{1} e^{i \zeta}$ and $\psi_{2}=\phi_{2} e^{i \zeta}$, for some real-valued nonnegative functions $\phi_{1}, \phi_{2}$ and $\zeta$. Then by computations it follows from $\Xi=0$ that

$(\beta-\gamma)\left(\phi_{1} \nabla \phi_{2}-\phi_{2} \nabla \phi_{1}\right)+2 i\left(r\left(\phi_{1} \nabla \phi_{2}-\phi_{2} \nabla \phi_{1}\right)+a(\nabla \zeta)\left(\phi_{1}^{2}-\phi_{2}^{2}\right)+(\gamma+\beta)(\nabla \zeta) \phi_{1} \phi_{2}\right)=0$

By taking the real part of the previous expression we get that $\phi_{1} \nabla \phi_{2}-\phi_{2} \nabla \phi_{1}=\phi_{1}^{2} \nabla\left(\phi_{2} / \phi_{1}\right)=$ 0 on $\Omega^{\prime}$, that is $\psi_{1}=\psi_{2}$ almost everywhere on $\Omega$, contradicting the linear independence of $\psi_{1}, \psi_{2}$. Then SAH2 is verified.

The proof of the second statement follows similar arguments and is thus omitted.

All the assumptions on $\mathcal{F}, \mathcal{A}, \mathcal{Y}$ in Theorem V.2 are satisfied, and in particular, for sufficiently small $I \subset \mathbb{R}$ and $\mathcal{U} \subset \mathcal{Y}$, we can define the manifolds $\mathcal{M}^{I, \mathcal{U}}$ of double eigenvalues.

Let us now consider controlled Hamiltonians in $\mathcal{F}$ of the kind

$$
\left(-i \nabla+\overline{\mathbf{A}}+u_{3} \mathbf{A}\right)^{2}+\bar{V}+u_{1} V_{1}+u_{2} V_{2}
$$

where $\bar{V}, V_{1}, V_{2} \in \mathcal{C}(\bar{\Omega}, \mathbb{R})$ and $\overline{\mathbf{A}}, \mathbf{A} \in \mathcal{C}^{1}\left(\bar{\Omega}, \mathbb{R}^{3}\right)$.

Lemma V.7 Let $(\bar{V}, \overline{\mathbf{A}}) \in \mathcal{Y}$ and $U$ as in Theorem V.5, and consider the map ev : $(\mathcal{C}(\bar{\Omega}, \mathbb{R}))^{2} \times \mathcal{C}^{1}\left(\bar{\Omega}, \mathbb{R}^{3}\right) \times U \rightarrow \mathcal{Y}$ defined as

$$
\mathrm{ev}\left(V_{1}, V_{2}, \mathbf{A}, \mathbf{u}\right)=\left(\bar{V}+u_{1} V_{1}+u_{2} V_{2}, \overline{\mathbf{A}}+u_{3} \mathbf{A}\right)
$$

Then ev is transversal to the manifolds $\mathcal{M}^{I, \mathcal{U}}$. 
Proof. Let us recall that $K(V, \mathbf{A})=(-i \nabla+\mathbf{A})^{2}+V$, and, in particular,

$$
K\left(\operatorname{ev}\left(V_{1}, V_{2}, \mathbf{A}, \mathbf{u}\right)\right)=\left(-i \nabla+\overline{\mathbf{A}}+u_{3} \mathbf{A}\right)^{2}+\bar{V}+u_{1} V_{1}+u_{2} V_{2}
$$

If $\operatorname{ev}\left(V_{1}, V_{2}, \mathbf{A}, \mathbf{u}\right) \notin \mathcal{M}^{I, \mathcal{U}}$ (that is, we recall, either $\operatorname{ev}\left(V_{1}, V_{2}, \mathbf{A}, \mathbf{u}\right) \notin \mathcal{U}$ or the Hamiltonian $H(\mathbf{u})=K\left(\operatorname{ev}\left(V_{1}, V_{2}, \mathbf{A}, \mathbf{u}\right)\right)$ has no double eigenvalues in the interval $\left.I\right)$, then the thesis trivially holds.

Assume then that $H(\overline{\mathbf{u}})=K\left(\operatorname{ev}\left(V_{1}, V_{2}, \mathbf{A}, \overline{\mathbf{u}}\right)\right)$ has a double eigenvalue $\lambda \in I$ for some $\overline{\mathbf{u}} \in U$ and $\left(V_{1}, V_{2}, \mathbf{A}\right)$ with $\operatorname{ev}\left(V_{1}, V_{2}, \mathbf{A}, \overline{\mathbf{u}}\right) \in \mathcal{U}$, and that $\psi_{1}, \psi_{2}$ are two orthonormal eigenstates of $H(\overline{\mathbf{u}})$ pertaining to $\lambda$. Without loss of generality, we assume that $\bar{u}_{1} \neq 0$.

Lemma V.6 ensures that the rank of the map

$$
(\delta V, \delta \mathbf{A}) \mapsto\left(f_{11}(\delta V, \delta \mathbf{A})-f_{22}(\delta V, \delta \mathbf{A}), f_{12}(\delta V, \delta \mathbf{A}), f_{21}(\delta V, \delta \mathbf{A})\right)
$$

where $f_{i j}(\delta V, \delta A)=\left\langle\psi_{i},\left(H_{\delta \mathbf{A}}+2 \mathbf{A} \cdot \delta \mathbf{A}+\delta V\right) \psi_{j}\right\rangle$, is three, and its restriction to the space $\{(\delta V, 0): \delta V \in \mathcal{C}(\bar{\Omega}, \mathbb{R})\}$ has rank at least two. Then, setting $\mathcal{K}(\delta \mathbf{A})=H_{\delta \mathbf{A}}+2\left(\overline{\mathbf{A}}+\bar{u}_{3} \mathbf{A}\right) \cdot \delta \mathbf{A}$, we can find three functions $\delta Z, \delta W \in \mathcal{C}(\bar{\Omega}, \mathbb{R})$ and $\delta \mathbf{A} \in \mathcal{C}^{1}\left(\bar{\Omega}, \mathbb{R}^{3}\right)$ such that the matrix

$$
\left(\begin{array}{ccc}
\left\langle\psi_{1}, \delta Z \psi_{2}\right\rangle & \left\langle\psi_{1}, \delta Z \psi_{2}\right\rangle^{*} & \left\langle\psi_{2}, \delta Z \psi_{2}\right\rangle-\left\langle\psi_{1}, \delta Z \psi_{1}\right\rangle \\
\left\langle\psi_{1}, \delta W \psi_{2}\right\rangle & \left\langle\psi_{1}, \delta W \psi_{2}\right\rangle^{*} & \left\langle\psi_{2}, \delta W \psi_{2}\right\rangle-\left\langle\psi_{1}, \delta W \psi_{1}\right\rangle \\
\left\langle\psi_{1}, \mathcal{K}(\delta \mathbf{A}) \psi_{2}\right\rangle & \left\langle\psi_{1}, \mathcal{K}(\delta \mathbf{A}) \psi_{2}\right\rangle^{*} & \left\langle\psi_{2}, \mathcal{K}(\delta \mathbf{A}) \psi_{2}\right\rangle-\left\langle\psi_{1}, \mathcal{K}(\delta \mathbf{A}) \psi_{1}\right\rangle
\end{array}\right)
$$

is nonsingular. In particular, by Proposition IV.3, $\mathbf{v}=0$ is a conical intersection for the Hamiltonian

$$
\widetilde{H}(\mathbf{v})=H(\overline{\mathbf{u}})+v_{1} \delta Z+v_{2} \delta W+v_{3} \mathcal{K}(\delta \mathbf{A})
$$

We now define the $\mathcal{C}^{1}$ map $\widetilde{q}: \mathbb{R}^{3} \rightarrow \mathcal{Y}$ as $\widetilde{q}(\mathbf{v})=\left(\bar{V}+\bar{u}_{1} V_{1}+\bar{u}_{2} V_{2}+v_{1} \delta Z+v_{2} \delta W, \overline{\mathbf{A}}+\right.$ $\left.\bar{u}_{3} \mathbf{A}+v_{3} \delta \mathbf{A}\right)$. It is easy to see that $K(\widetilde{q}(\mathbf{v}))=\widetilde{H}(\mathbf{v})$, and, in particular, $\mathbf{v}=0$ is a conical intersection for $K(\widetilde{q}(\mathbf{v}))$. Then, thanks to Proposition V.1, for every $\mathbf{v} \in \mathbb{R}^{3}$

$$
\widetilde{q}_{\mathbf{v}}=\sum_{j=1}^{3} v_{j} \partial_{j} \widetilde{q}(0)=\left(v_{1} \delta Z+v_{2} \delta W, v_{3} \delta \mathbf{A}\right)
$$

is not tangent to $\mathcal{M}^{I, \mathcal{U}}$. Since the codimension of $\mathcal{M}^{I, \mathcal{U}}$ in $\mathcal{Y}$ is three, then $\mathcal{Y}=\left\{\widetilde{q}_{\mathbf{v}}: \mathbf{v} \in\right.$ $\left.\mathbb{R}^{3}\right\}+T_{\widetilde{q}(0)} \mathcal{M}^{I, \mathcal{U}}$. 
To conclude the proof of the lemma it is enough to show that $\left\{\widetilde{q}_{\mathbf{v}}: \mathbf{v} \in \mathbb{R}^{3}\right\} \subset$ $\operatorname{Im}\left(\operatorname{ev}_{\left(V_{1}, V_{2}, \mathbf{A}, \overline{\mathbf{u}}\right)}^{\prime}\right)$. For this purpose we consider the variations in $(\mathcal{C}(\Omega, \mathbb{R}))^{2} \times \mathcal{C}^{1}\left(\bar{\Omega}, \mathbb{R}^{3}\right) \times \mathbb{R}^{3}$

$$
\begin{aligned}
& w_{1}=\left(\delta Z / \bar{u}_{1}, 0,0,0,0,0\right), \\
& w_{2}=\left(\delta W / \bar{u}_{1}, 0,0,0,0,0\right), \\
& w_{3}=\left(0,0, \delta \mathbf{A} / \bar{u}_{3}, 0,0,0\right),
\end{aligned}
$$

for which we have

$$
\begin{aligned}
\operatorname{ev}_{\left(V_{1}, V_{2}, \mathbf{A}, \overline{\mathbf{u}}\right)}^{\prime}\left(w_{1}\right) & =(\delta Z, 0), \\
\operatorname{ev}_{\left(V_{1}, V_{2}, \mathbf{A}, \overline{\mathbf{u}}\right)}^{\prime}\left(w_{2}\right) & =(\delta W, 0), \\
\operatorname{ev}_{\left(V_{1}, V_{2}, \mathbf{A}, \overline{\mathbf{u}}\right)}^{\prime}\left(w_{3}\right) & =(0, \delta \mathbf{A}) .
\end{aligned}
$$

This concludes the proof of the lemma.

We are ready to prove the main result of this section.

Proof of Theorem V.5. As in the proof of Theorem V.4, we first consider eigenvalues intersections occurring outside the origin. Namely, thanks to Lemma V.7, we can apply Theorem V.2 and conclude that, generically and for a prevalent subset of $(\mathcal{C}(\bar{\Omega}, \mathbb{R}))^{2} \times$ $\mathcal{C}^{1}\left(\bar{\Omega}, \mathbb{R}^{3}\right)$, all double eigenvalues of $H(\mathbf{u})$ with $\mathbf{u} \in U$ correspond to conical intersections.

Assume now that $\mathbf{u}=0$ is a double eigenvalues intersection for $H(\mathbf{u})$. Proceeding as in the proof of Theorem V.4 we have that $\mathbf{u}=0$ corresponds to a conical intersection if and only if $F\left(V_{1}, V_{2}, \mathbf{A}\right) \neq 0$, where

$$
F\left(V_{1}, V_{2}, \mathbf{A}\right)=\operatorname{det}\left(\begin{array}{ccc}
\mathfrak{R e}\left(\left\langle\psi_{1}, V_{1} \psi_{2}\right\rangle\right) & \mathfrak{I m}\left(\left\langle\psi_{1}, V_{1} \psi_{2}\right\rangle\right) & \left(\left\langle\psi_{2}, V_{1} \psi_{2}\right\rangle-\left\langle\psi_{1}, V_{1} \psi_{1}\right\rangle\right) \\
\mathfrak{R e}\left(\left\langle\psi_{1}, V_{2} \psi_{2}\right\rangle\right) & \mathfrak{I m}\left(\left\langle\psi_{1}, V_{2} \psi_{2}\right\rangle\right) & \left(\left\langle\psi_{2}, V_{2} \psi_{2}\right\rangle-\left\langle\psi_{1}, V_{2} \psi_{1}\right\rangle\right) \\
\mathfrak{R e}\left(\left\langle\psi_{1}, K_{\mathbf{A}} \psi_{2}\right\rangle\right) & \mathfrak{I m}\left(\left\langle\psi_{1}, K_{\mathbf{A}} \psi_{2}\right\rangle\right) & \left(\left\langle\psi_{2}, K_{\mathbf{A}} \psi_{2}\right\rangle-\left\langle\psi_{1}, K_{\mathbf{A}} \psi_{1}\right\rangle\right)
\end{array}\right),
$$

with $K_{\mathbf{A}}=H_{\mathbf{A}}+2 \overline{\mathbf{A}} \cdot \mathbf{A}$ and $\psi_{1}, \psi_{2}$ orthonormal eigenstates of $H(0)$ pertaining to the double eigenvalue. We have to show that the set $F^{-1}(\mathbb{R} \backslash\{0\})$ is both residual and prevalent in $(\mathcal{C}(\bar{\Omega}, \mathbb{R}))^{2} \times \mathcal{C}^{1}\left(\bar{\Omega}, \mathbb{R}^{3}\right)$.

We have that $F^{-1}(\mathbb{R} \backslash\{0\})$ is open. Density comes from Lemma V.6 (which in particular implies that $F$ is not identically zero) and multi-linearity of $F$. The set $F^{-1}(\mathbb{R} \backslash\{0\})$ is therefore residual.

To conclude the proof we pick $\bar{q} \in(\mathcal{C}(\bar{\Omega}, \mathbb{R}))^{2} \times \mathcal{C}^{1}\left(\bar{\Omega}, \mathbb{R}^{3}\right)$ such that $F(\bar{q}) \neq 0$ and we consider the measure $\mu$ on $(\mathcal{C}(\bar{\Omega}, \mathbb{R}))^{2} \times \mathcal{C}^{1}\left(\bar{\Omega}, \mathbb{R}^{3}\right)$ supported on the one-dimensional 
set $\{t \bar{q}: t \in[0,1]\}$ and induced by the Lebesgue measure on $[0,1]$. Since it is easy to see that, for any $q \in(\mathcal{C}(\bar{\Omega}, \mathbb{R}))^{2} \times \mathcal{C}^{1}\left(\bar{\Omega}, \mathbb{R}^{3}\right), F(q+t \bar{q})$ is a nonzero polynomial of degree three in the variable $t$ (having therefore at most three zeros on $[0,1]$ ), it turns out that $\mu\left(-q+F^{-1}(\mathbb{R} \backslash\{0\})\right)=1$. We thus obtain that $F^{-1}(\mathbb{R} \backslash\{0\})$ is prevalent. We conclude as in the proof of Theorem V.4.

\section{CONCLUSION}

In this paper we study the possibility that eigenvalues intersections for parameterdependent self-adjoint operators are conical. In particular we show that, for two important families of parameter-dependent Hamiltonians, the subfamilies of Hamiltonians admitting only conical intersections are both residual and prevalent. The main interest of this result relies on the fact that recent papers (in particular Ref. 9) have shown the possibility of exploiting the presence of conical intersection to obtain constructive approximate controllability results.

\section{ACKNOWLEDGMENTS}

This research has been supported by the project QUACO, PRC ANR-17-CE40-0007-01. F.C.C. has been partially supported by Fondation Sciences Mathématiques de Paris (FSMP) and by CARTT - IUT de Toulon.

\section{Appendix A: Regularity properties of perturbed self-adjoint operators}

In this section, we inspect some regularity properties of the spectrum and the spectral projections of parameter-dependent self-adjoint operators. Before focusing on operators satisfying the assumptions of Section II, we establish some regularity properties in a more general framework. Some of the results presented here are proved in Ref. 9 (by classical means, see also Ref. 19), therefore their proofs are omitted.

Here below, $\rho(A)$ and $\sigma(A)$ denote, respectively, the resolvent set and the spectrum of an operator $A$, and $R(A, \zeta)=(A-\zeta)^{-1}$ the resolvent of $A$ at $\zeta \in \mathbb{C}$.

We begin with the following technical lemma. 
Lemma A.1 $\left({ }^{9}\right)$ Let $A, B$ be two self-adjoint operators with $B$ A-bounded and $\zeta \in \rho(A)$. Then the following inequality holds

$$
\|B R(A, \zeta)\| \leq(1+(|\zeta|+1)\|R(A, \zeta)\|)\|B\|_{A} .
$$

In the following results, we analyze the behavior of the eigenvalues and the spectral projections under small perturbations of the operator. In particular, Lemma A.3 provides some estimates on the variation of the eigenvalues. For a similar result, see Ref. 19, Chapter 7, Theorem 3.6.

Lemma A.2 $\left({ }^{9}\right)$ Let $A_{1}$ be a self-adjoint operator and $I \subset \mathbb{R}$ be a nondegenerate and bounded interval whose boundary points belong to the resolvent set of $A_{1}$. Then for every $\epsilon>0$ there exists a $\delta>0$ such that, if $\left\|A_{1}-A_{2}\right\|_{A_{1}} \leq \delta$, then

i) $\sigma\left(A_{2}\right) \cap \partial I=\emptyset$ and if $\sigma\left(A_{1}\right) \cap I$ is made of $r$ eigenvalues, counted with multiplicity, then the same holds for $\sigma\left(A_{2}\right) \cap I$.

ii) Calling $P_{I}^{A_{1}}$ and $P_{I}^{A_{2}}$ the spectral projections on $I$ of $A_{1}$ and $A_{2}$, respectively, it holds

$$
\left\|P_{I}^{A_{1}}-P_{I}^{A_{2}}\right\| \leq \epsilon
$$

Lemma A.3 $\left({ }^{9}\right)$ Let $A_{1}$ be a self-adjoint operator and $I \subset \mathbb{R}$ be a nondegenerate, possibly unbounded, interval whose boundary points belong to the resolvent set of $A_{1}$. Assume that that $\sigma\left(A_{1}\right) \cap I$ is discrete and without finite accumulation points. If $\delta>0$ is small enough and $A_{2}$ is a self-adjoint operator satisfying $\left\|A_{2}-A_{1}\right\|_{A_{1}} \leq \delta$, then the eigenvalues of $A_{2}$ contained in $I$ are close to those of $A_{1}$, in the following sense. Up to appropriately indexing on a subset of $\mathbb{Z}$ the eigenvalues (counted with multiplicity) in $\sigma\left(A_{j}\right) \cap I$, for $j=1,2$, and denoting them with $\mu_{i}\left(A_{j}\right)$ we have $\left|\mu_{i}\left(A_{1}\right)-\mu_{i}\left(A_{2}\right)\right| \leq \epsilon\left(1+\left|\mu_{i}\left(A_{1}\right)\right|\right)$, where $\epsilon=e^{\frac{\delta}{1-\delta}}-1$.

For parameterized families of self-adjoint operators, we can prove some properties concerning the differentiability of the spectral projections associated with separated portion of the spectrum, as the following result shows (see also Refs. 19 and 30 for similar arguments).

Proposition A.4 Let $\mathcal{Y}$ be a Banach space with norm $\|\cdot\|_{\mathcal{Y}}$ and $K(\cdot)$ be a $\mathcal{C}^{1}$ function from $\mathcal{Y}$ to $\mathcal{B}_{\text {sa }}(\mathcal{D}(K(0)), \mathcal{H})$. Assume moreover that $K(q)-K(0)$ is $K(0)$-small for every $q \in \mathcal{Y}$. Then, for every $q_{0} \in \mathcal{Y}$ and every nondegenerate interval $I \subset \mathbb{R}$ whose boundary 
points belong to the resolvent set of $K\left(q_{0}\right)$, there exists a neighborhood $\mathcal{U}$ of $q_{0}$ in $\mathcal{Y}$ such that the spectral projection $P_{I}(q)$ on I associated with the self-adjoint operator $K(q)$ is well defined as a function from $\mathcal{U}$ to $\mathcal{B}_{\text {sa }}(\mathcal{H}, \mathcal{D}(K(0)))$ and $\mathcal{C}^{1}$ at $q_{0}$.

Proof. First of all, we recall that $K(\cdot)$ is $\mathcal{C}^{1}$ if it is Fréchet differentiable and its differential $q \mapsto K_{q}^{\prime}(\cdot)$, which takes values in the space of bounded linear operators from $\mathcal{Y}$ to $\mathcal{B}_{s a}(\mathcal{D}(K(0)), \mathcal{H})$, is continuous, that is, for every $\hat{q} \in \mathcal{Y}$

$$
\lim _{\|q-\hat{q}\|_{\mathcal{Y}} \rightarrow 0} \sup _{p \in \mathcal{Y}} \frac{\left\|K_{q}^{\prime}(p)-K_{\hat{q}}^{\prime}(p)\right\|_{K(0)}}{\|p\|_{\mathcal{Y}}}=0 .
$$

By hypothesis and Lemma A.2, there exists a closed path $\Gamma$ in $\mathbb{C}$ encircling $I$ such that, for $q$ in a small enough neighborhood $\mathcal{U}$ of $q_{0}$ all the elements of $\sigma(K(q))$ in the interior of $\Gamma$ belong to $I$. Then, $P_{I}(q)$ is given by Riesz formula

$$
P_{I}(q)=-(2 \pi i)^{-1} \oint_{\Gamma} R_{\zeta}(q) d \zeta
$$

where $R_{\zeta}(q)$ denotes the resolvent $R(K(q), \zeta)$. The boundedness of $P_{I}(q)$ in the norm $\|$. $\|_{\mathcal{B}(\mathcal{H}, \mathcal{D}(K(0)))}$ is a consequence of $(\mathrm{A} 1)$ and of the equivalence of the graph norms of $K(0)$ and $K(q)$.

Let us now prove Fréchet differentiability. We claim that the Fréchet differential of $P_{I}(q)$ at $q_{0}$ is given by the operator-valued function

$$
p \mapsto-(2 \pi i)^{-1} \oint_{\Gamma} R_{\zeta}\left(q_{0}\right) K_{q_{0}}^{\prime}(p) R_{\zeta}\left(q_{0}\right) d \zeta
$$

First of all we notice that, as a consequence of (A1), the integrand is bounded as an operator from $\mathcal{Y}$ to $\mathcal{B}(\mathcal{H}, \mathcal{D}(K(0)))$ and, since the corresponding bound varies continuously for $\zeta \in \Gamma$, the integral is bounded too. Take $\zeta$ belonging to the resolvent set of $K\left(q_{0}\right)$; thanks to (A1), possibly shrinking $\mathcal{U}$ we can write

$$
R_{\zeta}(q)=R_{\zeta}\left(q_{0}\right)\left(\mathrm{id}+\left(K(q)-K\left(q_{0}\right)\right) R_{\zeta}\left(q_{0}\right)\right)^{-1}=R_{\zeta}\left(q_{0}\right) \sum_{k=0}^{\infty}\left(\left(K\left(q_{0}\right)-K(q)\right) R_{\zeta}\left(q_{0}\right)\right)^{k}
$$

for every $q$ in $\mathcal{U}$. Then, from

$$
R_{\zeta}(q)-R_{\zeta}\left(q_{0}\right)-R_{\zeta}\left(q_{0}\right)\left(K\left(q_{0}\right)-K(q)\right) R_{\zeta}\left(q_{0}\right)=R_{\zeta}\left(q_{0}\right) \sum_{k=2}^{\infty}\left(\left(K\left(q_{0}\right)-K(q)\right) R_{\zeta}\left(q_{0}\right)\right)^{k}
$$

and since, possibly shrinking $\mathcal{U}$ again, we can assume that

$$
\left\|K(q)-K\left(q_{0}\right)\right\|_{K(0)} \leq 2\left\|K_{q_{0}}^{\prime}\right\|_{K(0)}\left\|q-q_{0}\right\|_{\mathcal{Y}}
$$


we have that there exists a constant $\hat{C}>0$, uniform with respect to $\zeta \in \Gamma$, such that

$$
\left\|R_{\zeta}(q)-R_{\zeta}\left(q_{0}\right)-R_{\zeta}\left(q_{0}\right)\left(K\left(q_{0}\right)-K(q)\right) R_{\zeta}\left(q_{0}\right)\right\| \leq \hat{C}\left\|q-q_{0}\right\|_{\mathcal{Y}}^{2}
$$

Setting $\Delta=R_{\zeta}\left(q_{0}+p\right)-R_{\zeta}\left(q_{0}\right)-R_{\zeta}\left(q_{0}\right) K_{q_{0}}^{\prime}(p) R_{\zeta}\left(q_{0}\right)$, we can write

$$
\|\Delta\|_{\mathcal{B}(\mathcal{H}, \mathcal{D}(K(0)))}=\|\Delta\|+\|K(0) \Delta\| .
$$

Splitting $K_{q_{0}}^{\prime}(p)$ as the sum of $K\left(q_{0}+p\right)-K\left(q_{0}\right)$ and $K_{q_{0}}^{\prime}(p)-K\left(q_{0}+p\right)+K\left(q_{0}\right)$, using the triangular inequality and applying (A2), we see that the first term in the right-hand side of (A3) is $o\left(\|p\|_{\mathcal{Y}}\right)$. The same computations plus the fact that $\left\|K(0) R_{\zeta}\left(q_{0}\right)\right\|$ is bounded prove that also the second term is $o\left(\|p\|_{\mathcal{Y}}\right)$. This proves the claim.

In order to prove the continuity of the differential of $P_{I}$ at $q_{0}$ it is enough to show the continuity of the operator-valued linear map $q \mapsto R_{\zeta}(q) K_{q}^{\prime}(\cdot) R_{\zeta}(q)$ at $q_{0}$, for $\zeta \in \Gamma$. This is a simple consequence of the continuity of $q \mapsto K_{q}^{\prime}(\cdot)$, the Second Resolvent Identity

$$
R_{\zeta}\left(q_{0}\right)-R_{\zeta}\left(q_{1}\right)=R_{\zeta}\left(q_{1}\right)\left(K\left(q_{1}\right)-K\left(q_{0}\right)\right) R_{\zeta}\left(q_{0}\right),
$$

and equation (A1).

Remark A.5 Let $\lambda(q)$ be a simple isolated eigenvalue of $K(q)$, for every $q$ in some domain $\mathcal{U} \subset \mathcal{Y}$. As a straightforward corollary of Proposition $A .4$ we obtain that $\lambda(\cdot)$ is $\mathcal{C}^{1}$ in $\mathcal{U}$. Analogously, there exists a $\mathcal{C}^{1}$ function $\phi: \mathcal{U} \rightarrow \mathcal{D}(K(0))$ such that $\phi(q)$ is an eigenstate of $K(q)$ corresponding to $\lambda(q)$, for every $q \in \mathcal{U}$.

We now focus on parameter-dependent self-adjoint operators that satisfy the hypotheses stated in Section II. In particular, in the next results $H: \mathbb{R}^{3} \rightarrow \mathcal{B}_{s a}(\mathcal{D}(H(0)), \mathcal{H})$ is a $\mathcal{C}^{1}$ map such that $H(\mathbf{u})-H(0)$ is $H(0)$-small for every $\mathbf{u}$.

Proposition A.6 Assume that, for $\mathbf{u}$ belonging to some bounded domain $U \subset \mathbb{R}^{3}$ and for some open bounded interval $I, \sigma(H(\mathbf{u})) \cap I$ is made of $m$ eigenvalues, counted with their multiplicity. Then $\sigma(H(\mathbf{u})) \cap I=\left\{\mu_{i}(\mathbf{u}): i=1, \ldots, m\right\}$, where every $\mu_{i}$ is Lipschitz continuous.

Proof. Applying the Mean-Value inequality, we get

$$
\left\|H(\mathbf{u})-H\left(\mathbf{u}^{\prime}\right)\right\|_{H(0)} \leq \sup _{t \in[0,1]}\left\|\nabla H\left(t \mathbf{u}+(1-t) \mathbf{u}^{\prime}\right)\right\|_{H(0)}\left|\mathbf{u}-\mathbf{u}^{\prime}\right|,
$$


where $\nabla H=\left(\partial_{1} H, \partial_{2} H, \partial_{3} H\right)$. Noticing that $\|\nabla H(\cdot)\|_{H(0)}$ is bounded on $U$, using the equivalence of the norms (1) and applying Lemma A.3, we get the thesis.

The last part of the section focuses on parametrized curves in the space of parameters; the regularity results proved here below concern the regularity with respect to $t$ of eigenvectors and eigenstates of $H(\mathbf{u}(t))$, for some sufficiently regular curve $\mathbf{u}: \mathbb{R} \rightarrow \mathbb{R}^{3}$.

Proposition A.7 Consider a $\mathcal{C}^{1}$ curve $\gamma:\left(t_{-}, t_{+}\right) \rightarrow \mathbb{R}^{3}$, with $t_{-}<t_{+}$, and assume that $\bar{\lambda}$ is a degenerate discrete eigenvalue of multiplicity $m$ of $H(\gamma(\bar{t}))$, for $\bar{t} \in\left(t_{-}, t_{+}\right)$. Then, possibly shrinking $\left(t_{-}, t_{+}\right)$around $\bar{t}$, there exist $m \mathcal{C}^{1}$ functions $\Lambda_{l}:\left(t_{-}, t_{+}\right) \rightarrow \mathbb{R}, l=1, \ldots, m$, such that $\Lambda_{l}(t)$ is an eigenvalue of $H(\gamma(t))$.

Proof. The proof is inspired from that of Ref. 28, Theorem XII.13. Thanks to Proposition A.6, we know that there exists an interval $I$ such that $\sigma(H(\mathbf{u})) \cap I$ is composed by $m$ eigenvalues, counted with multiplicity, for every $\mathbf{u}$ in a neighborhood $U$ of $\gamma(\bar{t})$; by Proposition A.4, the spectral projection $P_{I}(\mathbf{u})$ is $\mathcal{C}^{1}$ on $U$. For every $\mathbf{u} \in U$, we define the map $S(\mathbf{u}): P_{I}(\gamma(\bar{t})) \mathcal{H} \rightarrow P_{I}(\mathbf{u}) \mathcal{H}$ as

$$
S(\mathbf{u})=\left.P_{I}(\mathbf{u})\left(\mathrm{id}+P_{I}(\gamma(\bar{t}))\left(P_{I}(\mathbf{u})-P_{I}(\gamma(\bar{t}))\right) P_{I}(\gamma(\bar{t}))\right)^{-1 / 2}\right|_{P_{I}(\gamma(\bar{t})) \mathcal{H}}
$$

It is a $\mathcal{C}^{1}$ isometric transformation from the eigenspace relative to $\bar{\lambda}$ to the range of $P_{I}(\mathbf{u})$, which is the sum of the eigenspaces relative to the eigenvalues contained in $\sigma(H(\mathbf{u})) \cap I$ (see e.g. Ref. 30, Section 105). In particular, $t \mapsto S(\gamma(t))^{-1} H(\gamma(t)) S(\gamma(t))$ is a $\mathcal{C}^{1}$ family of symmetric $m$ dimensional operators acting on $P_{I}(\gamma(\bar{t})) \mathcal{H}$, having the same eigenvalues of $H(\gamma(t))$ in $I$. We can then apply Ref. 19, Theorem 6.8 and get the proof.

We remark that the smoothness of the operator is not sufficient to guarantee any regularity of the eigenstates and the eigenprojectors in presence of eigenvalues intersections, not even in the finite dimensional Hermitian case. Following Ref. 29, we consider the $\mathcal{C}^{\infty}$ matrix

$$
H(x)=e^{-1 / x^{2}}\left(\begin{array}{cc}
\cos (1 / x) & \sin (1 / x) \\
\sin (1 / x) & -\cos (1 / x)
\end{array}\right), \quad x \neq 0,
$$

with $H(0)=0$. Its eigenvalues are $\lambda(x)= \pm e^{-1 / x^{2}}$, therefore they are smooth, while its (normalized) eigenvectors are $\left(\begin{array}{c}\cos (1 /(2 x)) \\ \sin (1 /(2 x))\end{array}\right)$ and $\left(\begin{array}{c}-\sin (1 /(2 x)) \\ \cos (1 /(2 x))\end{array}\right)$, which do not have limit for $x \rightarrow 0$. 
If the difference between the intersecting eigenvalues grows linearly with respect to the parameters (as in the case of conical intersections), then the eigenvectors can be chosen continuous. This is the thesis of the following proposition (see also Ref. 15, Theorem 6 for a related result).

Proposition A.8 Let $\gamma:\left(t_{-}, t_{+}\right) \rightarrow \mathbb{R}^{3}$ be a $\mathcal{C}^{1}$ curve with $\dot{\gamma}(t) \neq 0$ for every $t \in\left(t_{-}, t_{+}\right)$, and assume that there exists an interval $I$ such that for every $t \in\left(t_{-}, t_{+}\right) \sigma(H(\gamma(t))) \cap I$ is composed by two eigenvalues $\lambda_{1}$ and $\lambda_{2}$. Assume moreover that there exists $\bar{t} \in\left(t_{-}, t_{+}\right)$such that $\lambda_{1}(\gamma(\bar{t}))=\lambda_{2}(\gamma(\bar{t}))$ and

$$
\left|\lambda_{2}(\gamma(t))-\lambda_{1}(\gamma(t))\right| \geq C|t-\bar{t}|
$$

for some $C>0$.

Then the projector $P_{i}(\gamma(\cdot))$ relative to $\lambda_{i}, i=1,2$, may be extended continuously on the whole $\left(t_{-}, t_{+}\right)$. Moreover, calling $H^{\operatorname{lin}}(t)=H(\gamma(\bar{t}))+(t-\bar{t}) \nabla H(\gamma(\bar{t})) \cdot \dot{\gamma}(t)$ the linearization of $H(\gamma(t))$ around $\bar{t}$, and $P_{1}^{\operatorname{lin}}(t), P_{2}^{\operatorname{lin}}(t)$ its projectors relative to the eigenvalues in $I$, we have that

$$
\lim _{t \rightarrow \bar{t}} P_{i}(\gamma(t))=\lim _{t \rightarrow \bar{t}} P_{i}^{\operatorname{lin}}(t), \quad i=1,2,
$$

where the limits above hold in the operator norm.

The proof of Proposition A.8 is a straightforward adaptation of that of Ref. 9, Proposition 3.1.

\section{REFERENCES}

${ }^{1}$ R. Abraham and J. Robbin. Transversal mappings and flows. An appendix by Al Kelley. W. A. Benjamin, Inc., New York-Amsterdam, 1967.

${ }^{2} \mathrm{R}$. Adami and U. Boscain. Controllability of the Schrödinger equation via intersection of eigenvalues. In Proceedings of the 44th IEEE Conference on Decision and Control, December 12-15, pages 1080-1085, 2005.

${ }^{3}$ A. A. Agrachev. Spaces of symmetric operators with multiple ground states. Funct. Anal. Appl., 45(4):241-251, 2011.

${ }^{4}$ N. Augier, U. Boscain, and M. Sigalotti. Adiabatic ensemble control of a continuum of quantum systems. SIAM Journal on Control and Optimization, pages 4045-4068, 2018. 
${ }^{5}$ G. Besson. Propriétés génériques des fonctions propres et multiplicité. Comment. Math. Helv., 64(4):542-588, 1989.

${ }^{6}$ U. Boscain, F. Chittaro, P. Mason, and M. Sigalotti. Adiabatic control of the Schrödinger equation via conical intersections of the eigenvalues. IEEE Trans. Automat. Control, 57(8):1970-1983, 2012.

${ }^{7}$ U. Boscain, J.-P. Gauthier, F. Rossi, and M. Sigalotti. Approximate controllability, exact controllability, and conical eigenvalue intersections for quantum mechanical systems. Communications in Mathematical Physics, 333(3):1225-1239, 2015.

${ }^{8}$ F.C. Chittaro and P. Mason. Approximate controllability by adiabatic methods of the Schrödinger equation with nonlinear hamiltonian. In Proceedings of the 54th IEEE Conference on Decision and Control, December 15-18, pages 7771-7776, 2015.

${ }^{9}$ F.C. Chittaro and P. Mason. Approximate controllability via adiabatic techniques for the three-inputs controlled Schrödinger equation. SIAM Journal on Control and Optimization, 55(6):4202-4226, 2017.

${ }^{10}$ Y. Colin De Verdière. Sur une hypothese de transversalité d'Arnold. Commentarii Mathematici Helvetici, 63(1):184-193, 1988.

${ }^{11}$ Yves Colin de Verdière. The level crossing problem in semi-classical analysis i. the symmetric case. In Annales de l'institut Fourier, volume 53, pages 1023-1054, 2003.

${ }^{12}$ Yves Colin de Verdière. The level crossing problem in semi-classical analysis. ii. the hermitian case. In Annales de l'institut Fourier, volume 54, pages 1423-1441, 2004.

${ }^{13}$ L. Dieci and A. Pugliese. Hermitian matrices depending on three parameters: Coalescing eigenvalues. Linear Algebra and its Applications, 436(11):4120 - 4142, 2012.

${ }^{14}$ W. Domcke, D. R. Yarkony, and H. Köppel. Conical Intersections. World Scientific, 2004. ${ }^{15}$ J.-P. Gauthier and V.M. Zakalyukin. On the codimension one motion planning problem. Journal of Dynamical and control systems, 11(1):73-89, 2005.

${ }^{16}$ G. A. Hagedorn. Molecular propagation through electron energy level crossings. Mem. Amer. Math. Soc., 111(536):vi+130, 1994.

${ }^{17}$ B. R. Hunt, T. Sauer, and J. A. Yorke. Prevalence: a translation-invariant "almost every" on infinite-dimensional spaces. Bulletin of the American mathematical society, 27(2):217238, 1992.

${ }^{18}$ R. Joly. Adaptation of the generic PDE's results to the notion of prevalence. Journal of Dynamics and Differential Equations, 19(4):967-983, Dec 2007. 
${ }^{19}$ T. Kato. Perturbation theory for linear operators. Die Grundlehren der mathematischen Wissenschaften, Band 132. Springer-Verlag New York, Inc., New York, 1966.

${ }^{20} \mathrm{~K}$. Kurata. A unique continuation theorem for the Schrödinger equation with singular magnetic field. Proceedings of the American Mathematical Society, pages 853-860, 1997.

${ }^{21}$ J. M Lee. Introduction to Smooth Manifolds. Springer, Second edition, 2013.

${ }^{22}$ D. Lupo and A.M. Micheletti. On multiple eigenvalues of self-adjoint compact operators. Journal of Mathematical Analysis and Applications, 172:106-116, 1993.

${ }^{23}$ Here and in the following all regularity assumptions rely on Fréchet differentiability; given two Banach spaces $X, Y$, the Fréchet differential of a differentiable function $f: X \rightarrow Y$ at $x \in X$ is denoted by $f_{x}^{\prime}$.

${ }^{24}$ In the community of quantum dynamics, the term conical intersection often refers to the case in which the eigenvalues cannot be made smooth around the intersection point by a simple reindexing. Note that in presence of a conical intersection in the sense of Definition III.1 the intersecting eigenvalues are always nonsmooth.

${ }^{25}$ While the previous notion of prevalence requires $\mathcal{A}$ to possess structure of vector space, the notion of residual set may be stated in the much more general context of Baire spaces. ${ }^{26}$ Similar examples may actually be found with $\Omega \subset \mathbb{R}^{d}$, for any $d \geq 2$, and indeed all the results in this section hold true if $d \geq 2$. The case $d=1$ is special. Indeed, on the one hand, it is easy to see that if $\Omega \subset \mathbb{R}$ and for every $\mathbf{A} \in \mathcal{C}^{1}(\bar{\Omega}, \mathbb{R})$, the energy levels of the Hamiltonian $-\partial_{x}^{2}+V+H_{\mathbf{A}}$ coincide with those of the Hamiltonian $-\partial_{x}^{2}+V-\mathbf{A}^{2}$, precluding the existence of conical intersections (the fact that for one dimensional systems a magnetic field can always be reabsorbed by an electric field is well known in physics). On the other hand it can be easily shown that Hamiltonians of the latter form, on bounded intervals and with Dirichlet boundary condition, do not admit degenerate eigenvalues.

${ }^{27}$ M. Reed and B. Simon. Methods of modern mathematical physics. II. Fourier analysis, self-adjointness. Academic Press [Harcourt Brace Jovanovich Publishers], New York, 1975.

${ }^{28} \mathrm{M}$. Reed and B. Simon. Methods of modern mathematical physics. IV. Analysis of operators. Academic Press [Harcourt Brace Jovanovich Publishers], New York, 1978.

${ }^{29}$ F. Rellich and J. Berkowitz. Perturbation Theory of Eigenvalue Problems. Notes on mathematics and its applications. Gordon and Breach, 1969.

${ }^{30}$ F. Riesz and B. Sz.-Nagy. Functional Analysis. Ungar, 1955.

${ }^{31}$ A.P. Seyranian, O.N. Kirillov, and A.A. Mailybaev. Coupling of eigenvalues of complex 
Conicity of eigenvalues intersections

matrices at diabolic and exceptional points. J. Phys. A: Math. Gen., 38:1723?1740, 2005.

${ }^{32}$ M. Teytel. How rare are multiple eigenvalues? Comm. Pure Appl. Math., 52(8):917-934, 1999.

${ }^{33}$ J. Von Neumann and E.P. Wigner. Über das Verhalten von Eigenwerten bei adiabatischen Prozessen. Physikalische Zeitschrift, 30:467-470, 1929. 